\title{
Sensitivity analysis of flexible multibody systems using composite materials components
}

\author{
Maria Augusta Neto ${ }^{1, * \dagger}$, Jorge A. C. Ambrósio ${ }^{2}$ and Rogério Pereira Leal ${ }^{1}$ \\ ${ }^{1}$ Departamento de Engenharia Mecânica-Faculdade de Ciência e Tecnologia da Universidade de Coimbra \\ (Polo II), 3020 Coimbra, Portugal \\ ${ }^{2}$ Instituto de Engenharia Mecânica-Instituto Superior Técnico, Av. Rovisco Pais, 1041-001 Lisboa, Portugal
}

\begin{abstract}
SUMMARY
In this paper, a general formulation for the computation of the first-order analytical sensitivities based on the direct method using automatic differentiation of flexible multibody systems is presented. The direct method for sensitivity calculation is obtained by differentiating the equations that define the response of the flexible multibody systems of composite materials with respect to the design variables, which are the ply orientations of the laminated. In order to appraise the benefits of the approach suggested and to highlight the risks of the procedure, the analytical sensitivities are compared with the numerical results obtained by using the finite difference method. For the beam composite material elements, the section properties and their sensitivities are found using an asymptotic procedure that involves a two-dimensional (2-D) finite element analysis of their cross section. The equations of the sensitivities are obtained by automatic differentiation and integrated in time simultaneously with the equations of motion of the multibody systems. The equations of motion and the sensitivities of the flexible multibody system are solved and the accelerations, velocities and the sensitivities of accelerations and velocities are integrated in time using a multi-step multi-order integration algorithm. Through the application of the methodology to two simple flexible multibody systems the difficulties and benefits of the procedure, with respect to finite difference approaches or to the direct implementation of the analytic sensitivities, are discussed. Copyright (C) 2008 John Wiley \& Sons, Ltd.
\end{abstract}

Received 12 January 2007; Revised 27 May 2008; Accepted 28 May 2008

KEY WORDS: elastic coupling; mode component synthesis; automatic differentiation; design sensitivity analysis

\footnotetext{
${ }^{*}$ Correspondence to: Maria Augusta Neto, Departamento de Engenharia Mecânica-Faculdade de Ciência e Tecnologia da Universidade de Coimbra (Polo II), 3020 Coimbra, Portugal.

†E-mail: augusta.neto@dem.uc.pt
} 


\section{INTRODUCTION}

Laminated fibrous composite materials are finding their way into a wide range of applications in structural design due to their superior mechanical properties, mainly for lightweight structures that have stringent stiffness and strength requirements. Designing with composite materials is a challenge due to the extensive range of parameters that can be selected. The best capable composite structural design of a given system that satisfies the requirements is achieved not only by selecting appropriate cross-sectional geometries but also by changing the number of layers, the orientation and the stacking sequence of lamina, which make up the composite laminate.

Many formalisms to describe the dynamics of flexible multibody systems have been proposed in the past, the most popular being the ones involving traditional finite element descriptions of the flexibility but having some new formulations, also based on finite elements but using absolute nodal coordinates as competing formulations [1]. The use of the flexible multibody dynamics in the framework of different areas has reported its use being in the analysis of flexible robots [2] or system identification [3] examples of new applications. The reduction in the complexity of the flexible multibody systems has also received attention due to the need for using more efficient models [4]. In all these areas and with all formulations and purposes the high potential of using composite materials optimized for the applications in sight is obvious.

The large set of design variables and the complex behavior of the composite materials make structural optimization an obvious tool for the design of more advanced structures. Optimization involves, in general, iterative schemes where the cost function and constraints are obtained in an analysis step and the sensitivities are computed to be used in the decision step of the optimization procedure. The computation of the sensitivity of a structural response to changes in design variables is often the major computational cost of the optimization process, and therefore efficient algorithms for evaluating these sensitivities are of fundamental importance.

The simplest procedure to calculate the sensitivity derivatives is the finite difference approximation [5]. This method is very popular mainly because of its easy implementation, which involves only the recursive use of the analysis program. However, the finite difference approximations may lead to problems resulting from the selection of the different step sizes used for the perturbations: Selection of a small step size may result in errors in the derivative due to round-off errors. Selection of a large step size can lead to truncation errors due to the neglected terms in the Taylor series expansion of the perturbed function [6]. Another drawback of this method concerns its poor numerical performance that results from requiring that additional analyses be performed for the perturbation of each additional design variables [7].

Analytical sensitivity formulations are alternatives for the exact evaluation of the derivatives of the dynamic response with respect to the design variables. In the work of Dias [8] the sensitivities of rigid-flexible multibody systems are based on the direct differentiation method and the sensitivity equations are obtained by a symbolic differentiation of the systems equation. When the direct differentiation is used, the integrations of the dynamic and sensitivity equations are all done at the same time; thus, the control of the time integration errors becomes more effective. In presence of a large number of potential design and state variables that may be used in the optimization of flexible multibody systems, the main disadvantage of the direct differentiation method is that it requires a large number of sensitivity equations to be derived analytically and implemented computationally, which involves cumbersome and prone to coding error procedures.

Recent developments in software technology have provided reliable automatic differentiation tools. Automatic differentiation is a technique for computing the derivatives of functions 
implemented in computer programs. ADIFOR [9] performs a transformation of functions, implemented computationally using Fortran 77, to obtain other functions also coded in the same language, which are the derivatives of the output of the first set of functions with respect to the design variables. The use of automatic differentiation results in a new computer code that provides accurate function gradients even for large and complex dynamic problems [10]. However, in what concerns the computational cost of this procedure with respect to either numerical derivatives or direct computer implementation of the analytical derivatives it is not always clear which one is the most efficient procedure. A discussion on the impact of using automatic differentiation on the cost of computing the derivatives is presented by Griewank and Reese [11]. ADIFOR is reported to be applied with success to obtain some terms in the sensitivity equation by Eberhard [12].

In this paper a general approach for the sensitivity analysis of rigid-flexible multibody systems with composite materials, based on the automatic differentiation method, is presented. The original code for the computation of the multibody rigid-flexible dynamic response, implemented in Fortran 77, is differentiated by using ADIFOR. In the process, the equilibrium equations for the flexible multibody systems made of composite materials and their analytic sensitivities are presented, to show which terms can be differentiated automatically and which analytic derivatives still have to be implemented by hand. The methodology is then applied to simple demonstrative examples to show its use and to support the discussion on its benefits and drawbacks.

\section{FLEXIBLE MULTIBODY SYSTEM DYNAMICS}

\subsection{Motion of a flexible body}

Let us assume that a flexible body is described in a coordinate system rigidly attached to a point on the flexible component, as depicted by Figure 1. Let us also assume that the deformation of the flexible body is represented using the finite element method, with a condensed representation of the mass matrix, obtained by the diagonalization of the original consistent mass matrix [13]. The reference frame located by vector $\mathbf{r}_{i}$ with respect to the inertia frame is fixed to the body center of mass [14]. The system equations of motion of this flexible body can be obtained by using Lagrange equations.

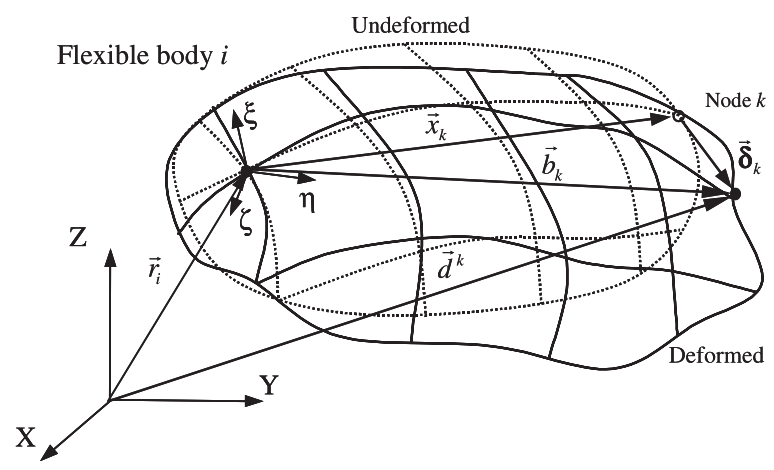

Figure 1. Global position of node $k$ in the flexible body. 
The flexible body kinetic energy is expressed as

$$
T_{i}=\frac{1}{2} \dot{\mathbf{q}}_{i}^{\mathrm{T}} \mathbf{M}_{i} \dot{\mathbf{q}}_{i}
$$

where the velocity vector for a single flexible body $i$ is represented by $\dot{\mathbf{q}}_{i}=\left[\begin{array}{lll}\dot{\mathbf{r}}_{i}^{\mathrm{T}} & \boldsymbol{\omega}_{i}^{\mathrm{T}} & \dot{\mathbf{u}}_{i}^{\prime}\end{array}\right]^{\mathrm{T}}$, which contains the body fixed frame translation velocities and the vector of the local nodal velocities $\dot{\mathbf{u}}_{i}^{\prime}=$ $\left[\dot{\boldsymbol{\delta}}^{\prime \mathrm{T}} \dot{\boldsymbol{\theta}}^{\prime \mathrm{T}}\right]_{i}^{\mathrm{T}} . \dot{\boldsymbol{\delta}}^{\prime}$ and $\dot{\boldsymbol{\theta}}^{\prime}$ are the vectors of the nodal translations and angular velocities, respectively, written with respect to the body fixed frame. The flexible body mass matrix $\mathbf{M}_{i}$ is given by

$$
\mathbf{M}_{i}=\left[\begin{array}{cccc}
\sum m_{k} \mathbf{I} & -\sum m_{k} \mathbf{A} \tilde{\mathbf{b}}_{k}^{\prime} & \sum m_{k} \mathbf{A} \underline{\mathbf{I}}_{k}^{\mathrm{T}} & \mathbf{0} \\
\sum m_{k} \tilde{\mathbf{b}}_{k}^{\prime} \mathbf{A}^{\mathrm{T}} & -\sum m_{k} \tilde{\mathbf{b}}_{k}^{\prime \mathrm{T}} \tilde{\mathbf{b}}_{k}^{\prime}+\sum \mu_{k} \mathbf{I} & \sum m_{k} \tilde{\mathbf{b}}_{k}^{\prime} \underline{\mathbf{I}}_{k}^{\mathrm{T}} & \sum \mu_{k} \underline{\mathbf{I}}_{k}^{\mathrm{T}} \\
\sum m_{k} \underline{\mathbf{I}}_{k} \mathbf{A}^{\mathrm{T}} & -\sum m_{k} \underline{\mathbf{I}}_{k} \tilde{\mathbf{b}}_{k}^{\prime} & \sum m_{k} \underline{\mathbf{I}}_{k} \underline{\mathbf{I}}_{k}^{\mathrm{T}} & \mathbf{0} \\
\mathbf{0} & \sum \mu_{k} \underline{\mathbf{I}}_{k} & \mathbf{0} & \sum \mu_{k} \underline{\mathbf{I}}_{k} \underline{\mathbf{I}}_{k}^{\mathrm{T}}
\end{array}\right]
$$

where $\mathbf{A}$ represents the transformation matrix from body fixed to inertial coordinates, $\mathbf{b}_{k}^{\prime}$ is the position of node $k$ with respect to the body fixed frame, $m_{k}$ is the nodal mass, $\mu_{k}$ is the nodal inertia, $\underline{\mathbf{I}}_{k}$ is a matrix filled with zeros except for the identity matrix associated with node $k$ and $(\approx)$ means the skew symmetric matrix associated with the quantity $(\cdot)$. For more details on the derivation of Equation (2) the reader is referred to [14].

When a consistent mass matrix is used in the finite element description of the flexible body, the inertia-coupling terms, represented by the off-diagonal sub-matrices in Equation (2), depend on the particular finite element shape functions used to formulate the finite elements. However, due to the diagonalization the structure of the mass matrix given by Equation (2) becomes independent of the formulation used to describe the flexible body deformations and no special inertia-coupling coefficients have to be derived with the purpose of using a particular finite element.

The elastic energy for the flexible body, expressed by $U$, is expressed as

$$
U=\frac{1}{2} \mathbf{q}^{\mathrm{T}} \underline{\mathbf{K}} \mathbf{q}
$$

where $\underline{\mathbf{K}}$ is an augmented stiffness matrix expressed as

$$
\underline{\mathbf{K}}=\left[\begin{array}{cc}
\mathbf{0} & \mathbf{0} \\
\mathbf{0} & \mathbf{K}_{f f}
\end{array}\right]
$$

$\mathbf{K}_{f f}$ being the standard finite element stiffness matrix of the flexible body. Let us assume that the formulation is valid only for small linear elastic deformations, which implies that matrix $\mathbf{K}_{f f}$ is not dependent on the deformation of the flexible body.

All coordinates defined in Equation (1) are independent, and therefore the Lagrange equations of motion for the flexible body can be expressed as

$$
\frac{\mathrm{d}}{\mathrm{d} t}\left(\frac{\partial T_{i}}{\partial \dot{\mathbf{q}}_{i}}\right)-\left(\frac{\partial T_{i}}{\partial \mathbf{q}_{i}}\right)+\left(\frac{\partial U_{i}}{\partial \mathbf{q}_{i}}\right)-\mathbf{g}_{i}=\mathbf{0}
$$

The kinetic energy and elastic energy, given by Equations (2) and (3), respectively, are now substituted in Equation (5) leading to

$$
\mathbf{M}_{i} \ddot{\mathbf{q}}_{i}=\mathbf{g}_{i}+\mathbf{s}_{i}-\underline{\mathbf{K}_{i}} \mathbf{q}_{i}
$$




\section{SENSITIVITY ANALYSIS OF FLEXIBLE MULTIBODY SYSTEMS}

In Equations (5) and (6) $\mathbf{g}_{i}$ is the vector of external applied forces and $\mathbf{s}_{i}$ is the vector of gyroscopic forces of the flexible body $i$.

\subsection{Equations of motion for a multibody system}

For a rigid and flexible multibody system, it is necessary to define a set of kinematic constraints that describe the joints used to restrict the relative motion between the bodies of the system. This implies that not all coordinates that describe the multibody system are independent. The kinematic constraints are mathematically defined by a set of algebraic equations that are added to the Lagrange equations by using a vector of Lagrange multipliers, $\lambda$. The equations of motion for a constrained multibody system are

$$
\frac{\mathrm{d}}{\mathrm{d} t}\left(\frac{\partial T}{\partial \dot{\mathbf{q}}}\right)-\left(\frac{\partial T}{\partial \mathbf{q}}\right)+\left(\frac{\partial U}{\partial \mathbf{q}}\right)+\mathbf{\Phi}_{\mathbf{q}}^{\mathrm{T}} \lambda-\mathbf{g}=\mathbf{0}
$$

which upon substitution of Equations (2) and (3) into Equation (7) leads to [15]

$$
\mathbf{M} \ddot{\mathbf{q}}+\boldsymbol{\Phi}_{\mathbf{q}}^{\mathrm{T}} \lambda=\mathbf{g}+\mathbf{s}-\underline{\mathbf{K}} \mathbf{q}
$$

where $\boldsymbol{\Phi}_{\mathbf{q}}$ is the Jacobian matrix of the kinematic constraints. The system of the equations of motion, defined by Equation (8), has more unknowns than independent equations. Therefore, to obtain its solution it is necessary to use the acceleration constraint equations also. The constraint acceleration equations, obtained as the second time derivatives of the position constraint equations, are given by

$$
\ddot{\mathbf{\Phi}}\left(\mathbf{q}_{r}, \mathbf{q}_{f}, t\right) \equiv \boldsymbol{\Phi}_{\mathbf{q}} \ddot{\mathbf{q}}-\boldsymbol{\gamma}=\mathbf{0}
$$

The Jacobian matrix can be partitioned into a rigid part $\boldsymbol{\Phi}_{\mathbf{q}_{r}}$ and a flexible part $\boldsymbol{\Phi}_{\mathbf{q}_{f}}$ such that $\boldsymbol{\Phi}_{\mathbf{q}}=\left[\begin{array}{ll}\boldsymbol{\Phi}_{\mathbf{q}_{r}}^{\mathrm{T}} & \boldsymbol{\Phi}_{\mathbf{q}_{f}}^{\mathrm{T}}\end{array}\right]^{\mathrm{T}}$. Substituting this expression into Equations (8) and (9) and writing them together lead to

$$
\left[\begin{array}{ccc}
\mathbf{M}_{r} & \mathbf{M}_{r f} & \boldsymbol{\Phi}_{\mathbf{q}_{r}}^{\mathrm{T}} \\
\mathbf{M}_{f r} & \mathbf{M}_{f f} & \boldsymbol{\Phi}_{\mathbf{q}_{f}}^{\mathrm{T}} \\
\boldsymbol{\Phi}_{\mathbf{q}_{r}} & \mathbf{\Phi}_{\mathbf{q}_{f}} & \mathbf{0}
\end{array}\right]\left\{\begin{array}{c}
\ddot{\mathbf{q}}_{r} \\
\ddot{\mathbf{u}}^{\prime} \\
\lambda
\end{array}\right\}=\left\{\begin{array}{c}
\mathbf{g}_{r} \\
\mathbf{g}_{f} \\
\boldsymbol{\gamma}
\end{array}\right\}-\left\{\begin{array}{c}
\mathbf{s}_{r} \\
\mathbf{s}_{f} \\
\mathbf{0}
\end{array}\right\}-\left\{\begin{array}{c}
\mathbf{0} \\
\mathbf{K}_{f f} \mathbf{u}^{\prime} \\
\mathbf{0}
\end{array}\right\}
$$

where the system accelerations are represented by vector $\ddot{\mathbf{q}}_{r}$, which contains the accelerations of the body fixed coordinate systems, and $\ddot{\mathbf{u}}^{\prime}$ is the vector of the generalized flexible coordinates, which groups the nodal accelerations of all flexible bodies in the system. The derivation of the kinematic joints involving one or more flexible bodies is, generally, a complex task that must be repeated for all different sets of flexible coordinates used. The virtual body technique, applied in the definition of the joints that involve flexible bodies, requires only that a single rigid joint is derived [14]. By attaching virtual bodies to all points of the flexible bodies where kinematic joints are applied the multibody model requires only standard kinematic joints between rigid bodies. This is the approach followed in all models presented in this work to avoid dealing with the formulation of particular kinematic joints involving flexible bodies.

The system of Equations (10) can be solved by applying any method suitable for the solution of linear algebraic equations. The existence of null elements in the main diagonal of the system matrix 
and the possibility of ill-conditioned matrices suggest that methods using partial or full pivoting are preferred. In this work, the subroutine dgesv from the LAPACK library [16], which performs the LU decomposition of the system matrix with partial pivoting and row interchanges, is used. The initial conditions related with the initial positions and velocities and the integration of the velocities and accelerations of the multibody system introduce numerical errors in the new positions and velocities obtained. These errors are due to the finite precision of the numerical methodologies and to the position and velocity constraint equations not appearing anywhere in the solution procedure. Therefore, methods able to eliminate errors in the constraint or velocity equations or, at least, to keep such errors under control are used. The Baumgarte stabilization method [17], in which feedback terms penalize the system response if violations on the position or velocity constraint equations occur, is the solution adopted in this work. This procedure requires that the right-hand side of Equation (9) is modified,

$$
\boldsymbol{\Phi}_{\mathbf{q}_{r}} \ddot{\mathbf{q}}_{r}+\boldsymbol{\Phi}_{\mathbf{q}_{f}} \ddot{\mathbf{u}}^{\prime}=\gamma-2 \alpha \dot{\boldsymbol{\Phi}}-\beta^{2} \boldsymbol{\Phi}
$$

where $\alpha$ and $\beta$ are positive constants that weight the violations of the velocity and position constraint equations, respectively. These constants, for a hybrid multibody system, are values in the range of $1-10, \alpha, \beta=1$ being the values often used [18]. For a comprehensive review of prior research on solution methods that handle constraints violations, the interest readers are referred to [19] and the references cited therein.

\subsection{Coordinate reduction by the component mode synthesis}

The equations of motion for flexible multibody systems, in the form described by Equation (10), lead to an inefficient numerical performance due to the large number of generalized flexible coordinates necessary to describe complex models. This problem is overcome by using a component mode synthesis methodology that effectively reduces the number of equations used to describe the flexible multibody system.

Let the nodal displacements of the flexible part of the body be described by a weighted sum of the modes of vibration associated with the flexible bodies' lower natural frequencies, given by

$$
\mathbf{u}^{\prime}=\mathbf{X} \mathbf{w}
$$

where vector $\mathbf{w}$ represents the contributions of the vibration modes towards the nodal displacements and $\mathbf{X}$ is the modal matrix. Owing to the reference conditions, which state that the body reference frame is fixed to the body center of mass, i.e. the relative displacements and rotations of the body center of mass with respect to the body frame are null, the modes of vibration used in this formulation are constrained modes. Moreover, due to the assumption of linear elastic deformations the modal matrix is invariant.

After substituting Equation (11) and its time derivatives into Equation (10) and using the orthonormality of the modes of vibration with respect to the mass matrix, i.e. $\mathbf{X}^{\mathrm{T}} \mathbf{M}_{f f} \mathbf{X}=\mathbf{I}$ and $\mathbf{X}^{\mathrm{T}} \mathbf{K}_{f f} \mathbf{X}=\boldsymbol{\Lambda}$, where $\boldsymbol{\Lambda}$ is a diagonal matrix containing the squares of the flexible body's natural frequencies, the equations of motion become

$$
\left[\begin{array}{ccc}
\mathbf{M}_{r} & \mathbf{M}_{r f} \mathbf{X} & \mathbf{\Phi}_{\mathbf{q}_{r}}^{\mathrm{T}} \\
\mathbf{X}^{\mathrm{T}} \mathbf{M}_{f r} & \mathbf{I} & \mathbf{X}^{\mathrm{T}} \boldsymbol{\Phi}_{\mathbf{q}_{f}}^{\mathrm{T}} \\
\boldsymbol{\Phi}_{\mathbf{q}_{r}} & \boldsymbol{\Phi}_{\mathbf{q}_{f}} \mathbf{X} & \mathbf{0}
\end{array}\right]\left\{\begin{array}{c}
\ddot{\mathbf{q}}_{r} \\
\ddot{\mathbf{w}} \\
\lambda
\end{array}\right\}=\left\{\begin{array}{c}
\mathbf{g}_{r} \\
\mathbf{X}^{\mathrm{T}} \mathbf{g}_{f} \\
\boldsymbol{\gamma}-2 \alpha \dot{\boldsymbol{\Phi}}-\beta^{2} \boldsymbol{\Phi}
\end{array}\right\}-\left\{\begin{array}{c}
\mathbf{s}_{r} \\
\mathbf{X}^{\mathrm{T}} \mathbf{s}_{f} \\
\mathbf{0}
\end{array}\right\}-\left\{\begin{array}{c}
\mathbf{0} \\
\boldsymbol{\Lambda} \mathbf{w} \\
\mathbf{0}
\end{array}\right\}
$$




\section{SENSITIVITY ANALYSIS OF FLEXIBLE MULTIBODY SYSTEMS}

Equation (12) represents a much smaller set of equations to describe the flexible bodies than that implied by Equation (10). Instead of having close to 6 times the number of the nodes of the finite element mesh as generalized flexible coordinates, the actual system only involves as many generalized flexible coordinates as the number of vibration modes used. Depending on the structure that is modeled only a few vibration modes may be used to represent with accuracy the structural response of each flexible body. All the terms required for Equation (12), related with a finite element model, are obtained directly from any finite element code. These coefficients are the finite element mass matrix $\mathbf{M}_{f f}$, the vibration modes used in the modal matrix $\mathbf{X}$ and the corresponding natural frequencies used in matrix $\boldsymbol{\Lambda}$. Equation (12) represents a linear system of equations for which the unknowns are the second-order time derivatives of generalized coordinates.

It should be noted that though only the modes of vibration associated with body coordinates systems fixed to the body center of mass are used in this procedure, other modes such as the static correction modes can also be used [20]. In the same manner, the use of the mean axis condition [21] could also be considered in order to improve numerical precision and the efficiency of the mode component synthesis.

\section{FLEXIBLE BODIES WITH COMPOSITE MATERIALS}

Laminated composite materials are effective substitutes for metallic materials in many structural applications. Though the analysis and design of these materials acquires an increasing importance in new constructive solutions, the design with composites is considerably more complex than the design with metallic materials. Certainly, a major difficulty in formulating the analysis of a laminated composite material results from its anisotropy. The characterization of the mechanical response of composite materials involves a number of material parameters substantially larger than what is required for metallic materials [22].

\subsection{Orthotropic lamina}

Composite materials consisting of stiff and strong fibers embedded in a compatible matrix are called fibrous composites. Laminated composites are made with layers of different materials that present various orientations. For a layer of orthotropic material, such as the one displayed in Figure 2, the material characteristics are described in three orthogonal planes $\left(\bar{x}_{1}, \bar{x}_{2}, \bar{x}_{3}\right)$ coinciding with the planes of material symmetry, which implies that the direction 3 of the global system and the direction 3 of the material systems are coincident, i.e. $\bar{x}_{3} \equiv x_{3}$. The constitutive equations are described, with respect to the frame $\left(\bar{x}_{1}, \bar{x}_{2}, \bar{x}_{3}\right)$ that forms the three orthogonal planes, as

$$
\left[\begin{array}{c}
\bar{\sigma}_{11} \\
\bar{\sigma}_{22} \\
\bar{\sigma}_{33} \\
\bar{\sigma}_{23} \\
\bar{\sigma}_{13} \\
\bar{\sigma}_{12}
\end{array}\right]_{k}\left(\left[\begin{array}{cccccc}
\bar{c}_{11} & \bar{c}_{12} & \bar{c}_{13} & 0 & 0 & 0 \\
\bar{c}_{21} & \bar{c}_{22} & \bar{c}_{23} & 0 & 0 & 0 \\
\bar{c}_{31} & \bar{c}_{32} & \bar{c}_{33} & 0 & 0 & 0 \\
0 & 0 & 0 & \bar{c}_{44} & 0 & 0 \\
0 & 0 & 0 & 0 & \bar{c}_{55} & 0 \\
0 & 0 & 0 & 0 & 0 & \bar{c}_{66}
\end{array}\right]\left[\begin{array}{c}
\bar{\varepsilon}_{11} \\
\bar{\varepsilon}_{22} \\
\bar{\varepsilon}_{33} \\
\bar{\varepsilon}_{23} \\
\bar{\varepsilon}_{13} \\
\bar{\varepsilon}_{12}
\end{array}\right]\right)_{k}
$$




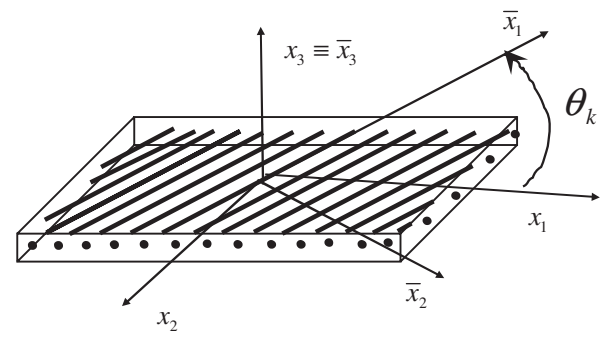

Figure 2. An orthotropic layer with principal material directions.

The elastic coefficients $\bar{c}_{i j}$ in Equation (13) are related to the material constants $E_{i}, v_{i j}$ and $G_{i j}$ as described in [22], and the index $k$ refers to the $k$ th layer.

Generally, the coordinate system used in the solution of the problem does not coincide with the material coordinate system. Furthermore, the composite laminates have several layers, each with a different orientation $\theta_{k}$ of their material coordinates with respect to the laminate coordinates. Thus, there is a need to establish a transformation that relates the stresses and strains in a given coordinate system with the corresponding quantities in another coordinate system. The relationship between $c_{i i}$ and the elastic coefficients in the material coordinates $\bar{c}_{i j}$ is given by

$$
\mathbf{C}_{k}=\left(\mathbf{T} \overline{\mathbf{C}} \mathbf{T}^{\mathrm{T}}\right)_{k}
$$

where $\mathbf{T}_{k}$ is the transformation matrix from the coordinate system associated with layer $k$ to the global coordinate frame.

Upon transformation, the lamina constitutive equations are expressed in the global coordinate system as

$$
\left[\begin{array}{c}
\sigma_{11} \\
\sigma_{22} \\
\sigma_{33} \\
\sigma_{23} \\
\sigma_{13} \\
\sigma_{12}
\end{array}\right]_{k}\left(\left[\begin{array}{cccccc}
c_{11} & c_{12} & c_{13} & 0 & 0 & c_{16} \\
c_{21} & c_{22} & c_{23} & 0 & 0 & c_{26} \\
c_{31} & c_{32} & c_{33} & c_{34} & c_{35} & c_{36} \\
0 & 0 & 0 & c_{44} & c_{45} & 0 \\
0 & 0 & 0 & c_{54} & c_{55} & 0 \\
c_{61} & c_{62} & c_{63} & 0 & 0 & c_{66}
\end{array}\right]\left[\begin{array}{c}
\varepsilon_{11} \\
\varepsilon_{22} \\
\varepsilon_{33} \\
2 \varepsilon_{23} \\
2 \varepsilon_{13} \\
2 \varepsilon_{12}
\end{array}\right]\right)_{k}
$$

where the $c_{i i}$ are the transformed elastic coefficients, which are now referred to the $\left(x_{1}, x_{2}, x_{3}\right)$ coordinate system.

\subsection{Stiffness of the composite plate/shell element}

The shell element used here is based on the Mindlin-Reissner plate theory, which requires only $C^{0}$ continuity for the approximation of the kinematic variables [23]. Using Hamilton's principle with the appropriate interpolation functions and following the standard finite displacement procedures, 


\section{SENSITIVITY ANALYSIS OF FLEXIBLE MULTIBODY SYSTEMS}

the equilibrium systems of linear equations that describe the flexible body with respect to the body fixed frame are

$$
\mathbf{M}_{f f} \ddot{\mathbf{q}}+\mathbf{K}_{f f} \mathbf{q}=\mathbf{F}
$$

At the element level, using the local coordinates $(\xi, \eta)$, the stiffness matrix is expressed as

$$
\mathbf{K}_{f f}^{(e)}=\int_{0}^{1} \int_{0}^{1-\eta}\left[\begin{array}{ccc}
\mathbf{B}_{m}^{\mathrm{T}} \mathbf{D}_{m} \mathbf{B}_{m} & \mathbf{B}_{m}^{\mathrm{T}} \mathbf{D}_{m b} \mathbf{B}_{b} & 0 \\
\mathbf{B}_{b}^{\mathrm{T}} \mathbf{D}_{b m} \mathbf{B}_{m} & \mathbf{B}_{b}^{\mathrm{T}} \mathbf{D}_{b} \mathbf{B}_{b} & 0 \\
0 & 0 & \mathbf{B}_{s}^{\mathrm{T}} \mathbf{D}_{s} \mathbf{B}_{s}
\end{array}\right]^{(e)}|\mathbf{J}| \mathrm{d} \xi \mathrm{d} \eta
$$

Equation (17) is expressed in a more compact form as

$$
\mathbf{K}_{f f}^{(e)}=\int_{0}^{1} \int_{0}^{1-\eta}\left(\mathbf{B}^{\mathrm{T}} \mathbf{D B}\right)^{(e)}|\mathbf{J}| \mathrm{d} \xi \mathrm{d} \eta
$$

where $\mathbf{B}$ is the strain-displacement matrix, $\mathbf{D}$ is the elasticity matrix and $|\mathbf{J}|$ is the determinant of the Jacobian matrix, which for triangular elements is twice the area of the element. The subscripts $m, b$ and $s$ stand for membrane, bending and shear, respectively. The strain-displacement matrix for the element is given by

$$
\mathbf{B}^{(e)}=\mathbf{L S}^{(e)}
$$

where $\mathbf{S}^{(e)}$ is the shape function matrix, which depends on the choice of the finite element and laminate theory, and $\mathbf{L}$ is a matrix of differential operators [24]. The sub-matrix $\mathbf{D}_{m}$ relates resultant membrane forces to membrane strains, the sub-matrix $\mathbf{D}_{b}$ relates generalized moments to generalized curvatures, the sub-matrix $\mathbf{D}_{m b}$ relates resultant membrane forces to generalized curvature and $\mathbf{D}_{s}$ relates resultant transverse shears to shear strains. As each layer of the laminate may have different properties, the elasticity matrix $\mathbf{D}$ is evaluated by a summation of the stiffness lamina over the thickness. Therefore, equivalent single layer theories produce equivalent stiffness matrices, which are weighted averages of the individual layer stiffness through the thickness. These matrices, which depend on each layer orientation, are given by

$$
\begin{aligned}
\left(\mathbf{D}_{m}, \mathbf{D}_{b}, \mathbf{D}_{m b}, \mathbf{D}_{s}\right)= & \sum_{k=1}^{n}\left(\mathbf{D}_{m}, \mathbf{D}_{b}, \mathbf{D}_{m b}, \mathbf{D}_{s}\right)_{k} \\
= & \sum_{k=1}^{n}\left(\left[\begin{array}{lll}
c_{11} & c_{12} & c_{16} \\
c_{21} & c_{22} & c_{26} \\
c_{61} & c_{62} & c_{66}
\end{array}\right] H_{1},\left[\begin{array}{lll}
c_{11} & c_{12} & c_{16} \\
c_{21} & c_{22} & c_{26} \\
c_{61} & c_{62} & c_{66}
\end{array}\right] H_{2},\right. \\
& {\left.\left.\left[\begin{array}{lll}
c_{11} & c_{12} & c_{16} \\
c_{21} & c_{22} & c_{26} \\
c_{61} & c_{62} & c_{66}
\end{array}\right] H_{3},\left[\begin{array}{ll}
c_{44} & c_{45} \\
c_{54} & c_{55}
\end{array}\right]\right)_{k}\right)_{k} }
\end{aligned}
$$

with

$$
H_{n}=\int_{h_{l-1}}^{h_{l}}\left(x_{3}^{n-1}\right) \mathrm{d} z=\frac{1}{n}\left(h_{l+1}^{n}-h_{l}^{n}\right)
$$




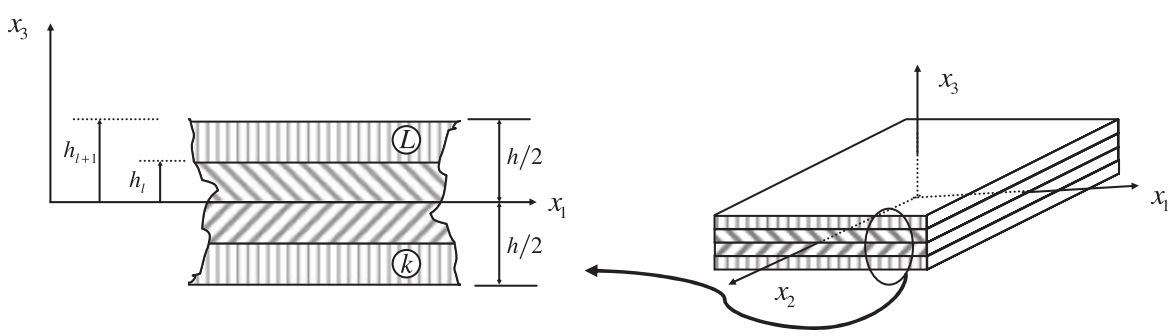

Figure 3. Coordinate system and layer numbering used for a typical laminated plate.

where $h_{l}$ is defined in Figure 3. The axis $x_{3}$ is defined to be positive upward from the mid-plane. The $l$ th layer is located between the points $x_{3}=h_{i}$ and $x_{3}=h_{i+1}$ in the thickness direction.

At the element level, and in local coordinates $(\xi, \eta)$, the consistent mass matrix is given by

$$
\mathbf{M}_{f f}^{(e)}=\int_{0}^{1} \int_{0}^{1-\eta} \rho^{(e)}\left(\mathbf{N}_{s}^{\mathrm{T}} \mathbf{m} \mathbf{N}_{s}\right)^{(e)}|\mathbf{J}| \mathrm{d} \xi \mathrm{d} \eta
$$

where $\mathbf{m}$ is the matrix with the inertia terms, $\mathbf{N}_{s}$ is the element shape matrix and $\rho$ is the specific mass of the element. Note that in the process of using the mass matrix in the representation of the flexible multibody formulation, a diagonalization procedure is used to obtain the lumped mass matrix [13].

The finite element developed is a triangular element with six degrees of freedom (d.o.f.) in each node, which include the nodal displacements $u_{1}^{0}, u_{2}^{0}, u_{3}^{0}, \phi_{1}, \phi_{2}$ and $\phi_{3}$. The rotation about the $x_{3}$ axis, $\phi_{3}$, is important in the case of shell elements and results from the projection of the other five d.o.f. in the global system [25].

\subsection{Stiffness matrix of the composite beam element}

In the finite element analysis of the 1-D beam problem the Timoshenko first-order shear deformation theory (FSDT) is used. In this theory, the transverse strains are constant through the depth of the beam; hence, the transverse shears are also constant. This discrepancy is overcome by introducing shear correction factors. For the beam element, at the element level and in local coordinates, the stiffness matrix is expressed as

$$
\mathbf{K}_{f f}^{(e)}=\int_{-1}^{1}\left(\mathbf{B}^{\mathrm{T}} \mathbf{D B}\right)^{(e)}|\mathbf{J}| \mathrm{d} \xi
$$

All the quantities involved in Equation (23) are defined in a form similar to those used in Equation (18). The $\mathbf{D}$ matrix is obtained using the code VABS, which is a general-purpose, 2-D finite-element-based cross-sectional analysis [26]. In the Timoshenko theory, matrix $\mathbf{D}$ can be expressed as

$$
\mathbf{D}=\left[\begin{array}{cc}
\mathbf{X} & \mathbf{F} \\
\mathbf{F}^{\mathrm{T}} & \mathbf{G}
\end{array}\right]
$$

where the sub-matrix $\mathbf{X}$ relates the resultant axial force $\mathbf{N}_{x x}$ and the generalized moments $\mathbf{M}=$ $\left[M_{y z}, M_{y y}, M_{z z}\right]^{\mathrm{T}}$ to the axial strain and the generalized curvatures. The sub-matrix $\mathbf{F}$ relates the 


\section{SENSITIVITY ANALYSIS OF FLEXIBLE MULTIBODY SYSTEMS}

resultant axial force and the generalized moments to the transverse shear, whereas the sub-matrix $\mathbf{G}$ relates the resultant transverse shear $\mathbf{Q}=2\left[\varepsilon_{12}, \varepsilon_{13}\right]^{\mathrm{T}}$ to the shear strains. These matrices are computed using a standard 2-D finite element code over the section of the beam. In the analysis of the beam cross section, the classical, $w_{0}$, and second-order warping, $w_{1}$, fields are discretized as

$$
\begin{aligned}
& w_{0}\left(x_{1}, x_{2}, x_{3}\right)=\mathbf{N}\left(x_{2}, x_{3}\right) \mathbf{V}_{0}\left(x_{1}\right) \\
& w_{1}\left(x_{1}, x_{2}, x_{3}\right)=\mathbf{N}\left(x_{2}, x_{3}\right) \mathbf{V}_{1}\left(x_{1}\right)
\end{aligned}
$$

where $\mathbf{N}$ represents the element shape functions and $\mathbf{V}_{0}$ and $\mathbf{V}_{1}$ are the column matrices with the nodal values of the warping displacements over the beam cross section. Using the variationalasymptotic method of Berdichevsky, the warping functions can be determined asymptotically in terms of 1-D strain measures as [27]

$$
\begin{aligned}
\mathbf{V}_{0} & =\hat{\mathbf{V}}_{0} \boldsymbol{\varepsilon} \\
\mathbf{E} \hat{\mathbf{V}}_{0} & =\left(\mathbf{H} \boldsymbol{\psi}_{c l} \boldsymbol{\psi}_{c l}^{\mathrm{T}}-\mathbf{I}\right) \mathbf{C}_{h \varepsilon}
\end{aligned}
$$

where $\boldsymbol{\varepsilon}$ are the generalized 1-D strain measures, $\hat{\mathbf{V}}_{0}$ is related to the discrete warping field, which is the solution of Equation (27), $\mathbf{H}$ is given by

$$
\mathbf{H}=\frac{1}{A} \int_{A} \mathbf{N}^{\mathrm{T}} \mathbf{N} \mathrm{d} A
$$

and $\psi_{c l}$ is related with the discrete form of the equations that remove the rigid-body modes from the warping field [28]. The second-order warping functions are computed using a procedure identical to that presented in Equations (27), i.e.

$$
\begin{aligned}
\mathbf{V}_{1} & =\hat{\mathbf{V}}_{1} \boldsymbol{\varepsilon}^{\prime} \\
\mathbf{E} \hat{\mathbf{V}}_{1} & =\left(\mathbf{I}-\mathbf{H} \boldsymbol{\psi}_{c l} \boldsymbol{\psi}_{c l}^{\mathrm{T}}\right)\left[\mathbf{C}_{\varepsilon l}^{\mathrm{T}}+\left(\mathbf{C}_{h l}^{\mathrm{T}}-\mathbf{C}_{h l}\right) \hat{\mathbf{V}}_{0}\right]
\end{aligned}
$$

where matrices $\mathbf{E}, \mathbf{C}_{h \varepsilon}, \mathbf{C}_{h l}, \mathbf{C}_{l l}$ and $\mathbf{C}_{g l}$ carry information on the material properties and on the geometry of a given beam cross section. For further details on the construction of these quantities, refer to [26].

The solution of Equations (27) and (29) are used in the strain energy. According to the procedure suggested by Yu et al. [26], the definition of matrix $\mathbf{G}$ is

$$
\mathbf{G}^{-1}=\mathbf{Q}^{\mathrm{T}} \mathbf{O}^{-\mathrm{T}} \mathbf{R} \mathbf{O}^{-1} \mathbf{Q}
$$

where matrices $\mathbf{Q}, \mathbf{O}$ and $\mathbf{R}$ are given by

$$
\begin{aligned}
& \mathbf{Q}=-\left[\begin{array}{cc}
0 & 0 \\
0 & 0 \\
0 & -1 \\
1 & 0
\end{array}\right], \quad \mathbf{O}=\mathbf{X}-\mathbf{F G}^{-1} \mathbf{F}^{\mathrm{T}} \\
& \mathbf{R}=\hat{\mathbf{V}}_{0}^{\mathrm{T}} \mathbf{C}_{l l} \hat{\mathbf{V}}_{0}+\hat{\mathbf{V}}_{1}^{\mathrm{T}}\left(\mathbf{C}_{h l}+\mathbf{C}_{h l}^{\mathrm{T}}\right) \hat{\mathbf{V}}_{0}+\hat{\mathbf{V}}_{1}^{\mathrm{T}} \mathbf{C}_{l \varepsilon}
\end{aligned}
$$


Matrix $\mathbf{F}$ is formulated as

$$
\mathbf{F}=\mathbf{B}^{\mathrm{T}} \mathbf{A}^{-1} \mathbf{Q G}, \quad \mathbf{B}=\left(\hat{\mathbf{V}}_{0}^{\mathrm{T}} \mathbf{C}_{h l}+\mathbf{C}_{\varepsilon l}^{\mathrm{T}}\right) \hat{\mathbf{V}}_{0}
$$

and matrices $\mathbf{X}$ and $\mathbf{A}$ are given by

$$
\mathbf{X}=\mathbf{A}+\mathbf{F G}^{-1} \mathbf{F}^{\mathrm{T}}, \quad \mathbf{A}=\mathbf{C}_{\varepsilon \varepsilon}+\hat{\mathbf{V}}_{0}^{\mathrm{T}} \mathbf{C}_{h \varepsilon}
$$

More details on the formulation of the composite beam element can be found in the work of $\mathrm{Yu}$ et al. [26].

\section{SENSITIVITY ANALYSIS IN MULTIBODY DYNAMICS}

Regardless of being used in the context of optimization or on its own, the sensitivity analysis requires reliable and accurate calculations of the sensitivities of different responses of a multibody system with respect to selected design parameters. Analytical sensitivities generally ensure that such accuracy is achieved. But, either because it is not always possible to obtain closed-form expressions for the sensitivity equations or because their implementation is too cumbersome their use is more limited than what would be expected. Consequently, the developer resorts to the use of numerical sensitivities that, under controlled conditions, provide an accuracy similar to that of the analytical sensitivities.

The use of automatic differentiation tools is an alternative to the closed-form derivation of analytical sensitivities. Automatic differentiation tools require that the complete analysis program be available in the source code. Then a new code that calculates the sensitivities is generated. The accuracy of the sensitivities calculated with the automatic differentiated code is similar to that of the analytical sensitivities. Unfortunately, not always the automatically differentiated tools provide reliable codes for the evaluation of the design sensitivities either because the some part of the source code of the analysis program is not available to the automatic differentiation tool or because the evaluation of the sensitivities involves more than just the analysis code. Yet, another source of difficulties results from the fact that the automatic differentiation tool differentiates more than just the equations of motion of the multibody system, i.e. it also differentiates all numerical procedures implemented such as numerical integrators, solvers of linear systems of equations or eigenproblem solvers.

In this work the automatic differentiation tool ADIFOR is used to derive the code used to evaluate the analytical sensitivities of flexible multibody systems made of composite materials. This new code is used in all calculations required to evaluate the sensitivities, except for the parts in which serious limitations are detected. In order to discuss some of the limitations of automatic differentiation tools, the analytical sensitivities for flexible multibody systems are fully derived here and the parts of the methodology not suitable for automatic differentiation are highlighted. Thus, the direct implementation of some of the numerical procedures, if necessary, complements the remaining code differentiated automatically. In addition, some parts of the multibody dynamic analysis program may not be available in the source code, as, for instance, the composite beam and plate elements. The closed-form equations for the analytical sensitivities of the composite material finite elements are provided for direct computer implementation if the interested reader cannot access the source code in which these elements are calculated. 


\section{SENSITIVITY ANALYSIS OF FLEXIBLE MULTIBODY SYSTEMS}

\subsection{Sensitivity analysis in the framework of optimization of multibody systems}

The sensitivity analysis aims at supplying the designer with quantitative information on how changes in the design variables influence the system performance. For a given design process a measure of its performance must be specified and, eventually, some design constraints must be defined. Typical objective functions used in multibody systems are mean values of the time response, extreme values of particular responses, integral accumulated values of state values or other derived quantities defined in an integral form. The types of optimization problems in multibody systems are extremely varied. Multibody optimal problems involving multiphysics [29], motion analysis of biomechanical systems [30,31], optimal tracking control [32] are just few examples of this diversity. For a given response of the dynamic system $f_{i}(\mathbf{b}, \mathbf{z}, \boldsymbol{\lambda}, t)$ a typical objective function can be defined as

$$
\psi_{i}=\int_{t_{i}}^{t_{e}} f_{i}(\mathbf{b}, \mathbf{z}, \lambda, t) \mathrm{d} t, \quad i=0, \ldots, n
$$

where the state vector $\mathbf{z}$ includes the coordinates, velocities and accelerations of the system, which depend on time and on the design parameters, denoted by $\mathbf{b}$. This dependency can be expressed as

$$
\mathbf{z}(\mathbf{b}, t)=(\mathbf{q}(\mathbf{b}, t), \dot{\mathbf{q}}(\mathbf{b}, t), \ddot{\mathbf{q}}(\mathbf{b}, t))
$$

The goal of the sensitivity analysis is to compute the derivative of all objective and constraints functions with respect to the design variables. For a set of design variables the derivatives are the gradients of the functions defined as

$$
\nabla \boldsymbol{\psi}_{i}=\frac{\mathrm{d} \boldsymbol{\psi}_{i}}{\mathrm{db}}, \quad i=0, \ldots, n
$$

These derivatives are calculated by using the chain rule as

$$
\frac{\mathrm{d} \psi_{i}}{\mathrm{~d} \mathbf{b}}=\frac{\partial \psi_{i}}{\partial \mathbf{b}}+\frac{\partial \psi_{i}}{\partial \mathbf{q}} \frac{\partial \mathbf{q}}{\partial \mathbf{b}}+\frac{\partial \psi_{i}}{\partial \dot{\mathbf{q}}} \frac{\partial \dot{\mathbf{q}}}{\partial \mathbf{b}}+\frac{\partial \psi_{i}}{\partial \ddot{\mathbf{q}}} \frac{\partial \ddot{\mathbf{q}}}{\partial \mathbf{b}}+\frac{\partial \psi_{i}}{\partial \lambda} \frac{\partial \boldsymbol{\lambda}}{\partial \mathbf{b}}
$$

In a more compact notation, the partial derivatives of the state variables are represented as

$$
\frac{\mathrm{d} \psi_{i}}{\mathrm{~d} \mathbf{b}}=\frac{\partial \psi_{i}}{\partial \mathbf{b}}+\frac{\partial \psi_{i}}{\partial \mathbf{q}} \mathbf{q}_{\mathbf{b}}+\frac{\partial \psi_{i}}{\partial \dot{\mathbf{q}}} \dot{\mathbf{q}}_{\mathbf{b}}+\frac{\partial \psi_{i}}{\partial \ddot{\mathbf{q}}} \ddot{\mathbf{q}}_{\mathbf{b}}+\frac{\partial \psi_{i}}{\partial \boldsymbol{\lambda}} \boldsymbol{\lambda}_{\mathbf{b}}
$$

When evaluating Equation (39) the computations associated with the terms $\partial \psi_{i} / \partial \mathbf{b}, \partial \psi_{i} / \partial \mathbf{q}$, $\partial \psi_{i} / \partial \dot{\mathbf{q}}, \partial \psi_{i} / \partial \ddot{\mathbf{q}}$ and $\partial \psi_{i} / \partial \lambda$ are very simple due to the explicit dependency of Equation (35) on the design and state variables. Therefore, these derivatives are obtained analytically and implemented directly in the computer code. The coding of the sensitivities of the state variables with respect to the design variables is rather complex and, if done explicitly, prone to implementation errors. However, by using automatic differentiation this task becomes much simpler and robust by safely avoiding programming errors while preserving the precision of the analytic derivatives. Therefore, all derivatives of the state variables and Lagrange multipliers with respect to the design variables, used in this work, are obtained by applying an automatic differentiation tool. 


\subsection{Sensitivities of the equation of motion}

For a flexible multibody system the modal form of the equations of motion is given by Equation (12). To calculate the sensitivities of the state variables with respect to the design variables, this equation is differentiated with respect to the design variables $\mathbf{b}$ and rearranged as

$$
\left[\begin{array}{ccc}
\mathbf{M}_{r} & \mathbf{M}_{r f} \mathbf{X} & \boldsymbol{\Phi}_{\mathbf{q}_{r}}^{\mathrm{T}} \\
\mathbf{X}^{\mathrm{T}} \mathbf{M}_{f r} & \mathbf{I} & \mathbf{X}^{\mathrm{T}} \boldsymbol{\Phi}_{\mathbf{q}_{f}}^{\mathrm{T}} \\
\boldsymbol{\Phi}_{\mathbf{q}_{r}} & \boldsymbol{\Phi}_{\mathbf{q}_{f}} \mathbf{X} & \mathbf{0}
\end{array}\right]\left\{\begin{array}{l}
\ddot{\mathbf{q}}_{r_{\mathbf{b}}} \\
\ddot{\mathbf{w}}_{\mathbf{b}} \\
\boldsymbol{\lambda}_{\mathbf{b}}
\end{array}\right\}=\left\{\begin{array}{c}
\mathbf{Q}_{\mathbf{b}} \\
\mathbf{R}_{\mathbf{b}} \\
\gamma_{\mathbf{b}}-2 \alpha \dot{\boldsymbol{\Phi}}_{\mathbf{b}}-\beta^{2} \boldsymbol{\Phi}_{\mathbf{b}}
\end{array}\right\}
$$

where $\mathbf{Q}_{\mathbf{b}}, \mathbf{R}_{\mathbf{b}}$ and $\gamma_{\mathbf{b}}$ are given by

$$
\begin{aligned}
& \mathbf{Q}_{\mathbf{b}}=\frac{\partial}{\partial \mathbf{q}_{r}}\left(\mathbf{g}_{r}-\mathbf{s}_{r}-\mathbf{M}_{r} \hat{\ddot{\mathbf{q}}}_{r}-\mathbf{M}_{r f} \mathbf{X} \hat{\ddot{\mathbf{w}}}-\mathbf{\Phi}_{\mathbf{q}_{r}}^{\mathrm{T}} \hat{\lambda}\right) \mathbf{q}_{r_{\mathbf{b}}}+\frac{\partial}{\partial \dot{\mathbf{q}}_{r}}\left(\mathbf{g}_{r}-\mathbf{s}_{r}\right) \dot{\mathbf{q}}_{r_{\mathbf{b}}} \\
& +\frac{\partial}{\partial \mathbf{w}}\left(\mathbf{g}_{r}-\mathbf{s}_{r}-\mathbf{M}_{r} \hat{\hat{\mathbf{q}}}_{r}-\mathbf{M}_{r f} \mathbf{X} \hat{\hat{\mathbf{w}}}-\boldsymbol{\Phi}_{\mathbf{q}}^{\mathrm{T}} \hat{\boldsymbol{\lambda}}\right) \mathbf{w}_{\mathbf{b}}+\frac{\partial}{\partial \dot{\mathbf{w}}}\left(\mathbf{g}_{r}-\mathbf{s}_{r}\right) \dot{\mathbf{w}}_{\mathbf{b}} \\
& +\frac{\partial}{\partial \mathbf{b}}\left(\mathbf{g}_{r}-\mathbf{s}_{r}-\mathbf{M}_{r} \hat{\ddot{\mathbf{q}}}_{r}-\mathbf{M}_{r f} \mathbf{X} \hat{\ddot{\mathbf{w}}}-\boldsymbol{\Phi}_{\mathbf{q}_{r}^{\mathrm{T}}}^{\hat{\lambda}}\right) \\
& \mathbf{R}_{\mathbf{b}}=\frac{\partial}{\partial \mathbf{q}_{r}}\left(\mathbf{X}^{\mathrm{T}} \mathbf{g}_{f}-\mathbf{X}^{\mathrm{T}} \mathbf{s}_{f}-\mathbf{X}^{\mathrm{T}} \mathbf{K}_{f f} \mathbf{X} \mathbf{w}-\mathbf{X}^{\mathrm{T}} \mathbf{M}_{f r} \hat{\ddot{\mathbf{q}}}_{r}-\mathbf{X}^{\mathrm{T}} \mathbf{M}_{f f} \mathbf{X} \hat{\ddot{\mathbf{w}}}-\mathbf{X}^{\mathrm{T}} \mathbf{\Phi}_{\mathbf{q}_{f}^{\mathrm{T}}}^{\mathrm{\lambda}}\right) \mathbf{q}_{r_{\mathbf{b}}} \\
& +\frac{\partial}{\partial \mathbf{w}}\left(\mathbf{X}^{\mathrm{T}} \mathbf{g}_{f}-\mathbf{X}^{\mathrm{T}} \mathbf{s}_{f}-\mathbf{X}^{\mathrm{T}} \mathbf{K}_{f f} \mathbf{X} \mathbf{w}-\mathbf{X}^{\mathrm{T}} \mathbf{M}_{f r} \hat{\ddot{\mathbf{q}}}_{r}-\mathbf{X}^{\mathrm{T}} \mathbf{M}_{f f} \mathbf{X} \hat{\ddot{\mathbf{w}}}-\mathbf{X}^{\mathrm{T}} \mathbf{\Phi}_{\mathbf{q}_{f}^{\mathrm{T}}}^{\hat{\lambda}}\right) \mathbf{w}_{\mathbf{b}} \\
& +\frac{\partial}{\partial \mathbf{b}}\left(\mathbf{X}^{\mathrm{T}} \mathbf{g}_{f}-\mathbf{X}^{\mathrm{T}} \mathbf{s}_{f}-\mathbf{X}^{\mathrm{T}} \mathbf{K}_{f f} \mathbf{X} \mathbf{w}-\mathbf{X}^{\mathrm{T}} \mathbf{M}_{f r} \hat{\ddot{\mathbf{q}}}_{r}-\mathbf{X}^{\mathrm{T}} \mathbf{M}_{f f} \mathbf{X} \hat{\hat{\mathbf{w}}}-\mathbf{X}^{\mathrm{T}} \boldsymbol{\Phi}_{\mathbf{q}_{f}^{\mathrm{T}}}^{\hat{\boldsymbol{\lambda}}}\right) \\
& +\frac{\partial}{\partial \dot{\mathbf{q}}_{r}}\left(\mathbf{X}^{\mathrm{T}} \mathbf{g}_{f}-\mathbf{X}^{\mathrm{T}} \mathbf{s}_{f}\right) \dot{\mathbf{q}}_{r_{\mathbf{b}}}+\frac{\partial}{\partial \dot{\mathbf{w}}}\left(\mathbf{X}^{\mathrm{T}} \mathbf{g}_{f}-\mathbf{X}^{\mathrm{T}} \mathbf{s}_{f}\right) \dot{\mathbf{w}}_{\mathbf{b}} \\
& \gamma_{\mathbf{b}}=\frac{\partial}{\partial \mathbf{q}_{r}}\left(\boldsymbol{\gamma}-\boldsymbol{\Phi}_{\mathbf{q}_{r}} \hat{\ddot{\mathbf{q}}}_{r}-\boldsymbol{\Phi}_{\mathbf{q}_{f}} \mathbf{X} \hat{\ddot{\mathbf{w}}}\right) \mathbf{q}_{r_{\mathbf{b}}}+\frac{\partial}{\partial \mathbf{w}}\left(\gamma-\boldsymbol{\Phi}_{\mathbf{q}_{r}} \hat{\ddot{\mathbf{q}}}_{r}-\boldsymbol{\Phi}_{\mathbf{q}_{f}} \mathbf{X} \hat{\ddot{\mathbf{w}}}\right) \mathbf{w}_{\mathbf{b}} \\
& +\frac{\partial}{\partial \mathbf{b}}\left(\boldsymbol{\gamma}-\boldsymbol{\Phi}_{\mathbf{q}_{r}} \hat{\ddot{\mathbf{q}}}_{r}-\boldsymbol{\Phi}_{\mathbf{q}_{f}} \mathbf{X} \hat{\ddot{\mathbf{w}}}\right)+\frac{\partial \gamma}{\partial \dot{\mathbf{q}}_{r}} \dot{\mathbf{q}}_{r_{\mathbf{b}}}+\frac{\partial \gamma}{\partial \dot{\mathbf{w}}} \dot{\mathbf{w}}_{\mathbf{b}}
\end{aligned}
$$

The superscript $(\hat{*})$ is used to denote that the quantity $(\cdot)$ remains constant during the differentiation process. Equation (40), as well as Equation (12) before, represents a linear system of equations for which the unknowns are the sensitivities of the second-order time derivatives of generalized coordinates. These linear systems of equations, which share the same leading matrix, are solved using the LU factorization scheme [16].

It must be emphasized here that all terms appearing in the right-hand side of Equation (40) are obtained by the automatic differentiation of the code, in which the system equations of motion are implemented, except for the parts of the sensitivities that involve the modes of vibration, which are discussed in Section 4.4, and, eventually, the sensitivities of the composite material finite 


\section{SENSITIVITY ANALYSIS OF FLEXIBLE MULTIBODY SYSTEMS}

element matrices, discussed in Sections 4.5 and 4.6, if the source code used for their evaluation is not available.

\subsection{Integration of the time derivatives of the sensitivities}

The solution of Equation (40) yields the time derivatives of the state variables' sensitivities with respect to the design variables, written as $\ddot{\mathbf{q}}_{r_{\mathbf{b}}}$ and $\ddot{\mathbf{w}}_{\mathbf{b}}$. The sensitivities of the state variables associated with the position and orientation of the body fixed coordinate frames $\mathbf{q}_{r_{\mathbf{b}}}$ and with the modal coordinates $\mathbf{w}_{\mathbf{b}}$, and their time derivatives, are obtained by direct integration of $\dot{\mathbf{q}}_{r_{\mathbf{b}}}, \dot{\mathbf{w}}_{\mathbf{b}}, \ddot{\mathbf{q}}_{r_{\mathbf{b}}}, \ddot{\mathbf{w}}_{\mathbf{b}}$. At this point the similarity between Equations (40) and (12) should be noted. Both are linear systems of equations with equal leading matrices. Therefore, by a proper choice of the solution method, the entire computational burden can be put in the solution of Equation (12), the solution of Equation (40) being obtained inexpensively.

The integration process is started by providing the initial conditions of the sensitivities as

$$
\begin{aligned}
\mathbf{q}_{r_{\mathbf{b}}}\left(t_{0}\right) & =\mathbf{q}_{r_{\mathbf{b}}}^{0} \\
\mathbf{w}_{\mathbf{b}}\left(t_{0}\right) & =\mathbf{w}_{\mathbf{b}}^{0} \\
\dot{\mathbf{q}}_{r_{\mathbf{b}}}\left(t_{0}\right) & =\dot{\mathbf{q}}_{r_{\mathbf{b}}}^{0} \\
\dot{\mathbf{w}}_{\mathbf{b}}\left(t_{0}\right) & =\dot{\mathbf{w}}_{\mathbf{b}}^{0}
\end{aligned}
$$

Equation (12) is solved to obtain the system accelerations and the factorized leading matrix retained to be used in the solution of Equation (40). However, due to the automatic differentiation tool the subroutine that computes the solution of the system equations of motion is differentiated to obtain the sensitivity of the solution vector. The differentiated version of the subroutine not only computes the new quantities, which are the sensitivities of the solution vector, but also preserves the computation of the previous quantities, which are the system accelerations. Therefore, the solution of Equation (12), for $\ddot{\mathbf{q}}_{r}$ and $\ddot{\mathbf{w}}$, and the solution of Equation (40), for $\ddot{\mathbf{q}}_{r_{\mathbf{b}}}$ and $\ddot{\mathbf{w}}_{\mathbf{b}}$, are in fact obtained simultaneously. This is certainly one of the major differences between using a traditional approach based on the implementation of analytic sensitivities and the use of automatic differentiation.

In the process of the automatic differentiation of the analysis code not only the routines used to formulate the equations of motion are differentiated but also the numerical integrator algorithm, used in their solution, is differentiated. If the differentiated integrator is used to integrate the state variables $\dot{\mathbf{q}}_{r}, \dot{\mathbf{w}}, \ddot{\mathbf{q}}_{r}, \ddot{\mathbf{w}}$ and their sensitivities $\dot{\mathbf{q}}_{r_{\mathbf{b}}}, \dot{\mathbf{w}}_{\mathbf{b}}, \ddot{\mathbf{q}}_{r_{\mathbf{b}}}, \ddot{\mathbf{w}}_{\mathbf{b}}$, wrong results are obtained [33]. Even if the automatic differentiation of the integrator is correct its step size control is based only on the local error estimative for the state variables and not on the local errors of the estimates of their gradients. Therefore, the code that is offered to the automatic differentiation tool includes the numerical integrator DE/STEP by Shampine and Gordon [34], for which the source code is available. After a differentiated code is obtained, the original integrator is substituted by another numerical integrator that uses the gear method, as implemented in the IMSL library [35]. The state variables and the sensitivities are included in the same vector that is to be integrated. Consequently, all variables are integrated with the same time step and the same error control.

After the direct integration of the equations of motion the constraint equations and, even the constraint equations' sensitivities, may not be fulfilled due to the limited numerical precision or due to integration errors. The Baumgarte stabilization procedure that modifies the right-hand side 
of the acceleration constraint equations is applied to handle the sensitivity constraint violations as well. The right-hand side of the last row of Equation (40) is now

$$
\boldsymbol{\Phi}_{\mathbf{q}_{\mathbf{b}}} \ddot{\mathbf{q}}_{r_{\mathbf{b}}}+\boldsymbol{\Phi}_{\mathbf{q}_{f}} \mathbf{X} \ddot{\mathbf{w}}_{\mathbf{b}}=\gamma_{\mathbf{b}}-2 \alpha \dot{\boldsymbol{\Phi}}_{\mathbf{b}}-\beta^{2} \mathbf{\Phi}_{\mathbf{b}}
$$

The terms that multiply by $\alpha$ and $\beta$ in equation are introduced as error feedbacks for the sensitivities of the acceleration constraint equations that use the violations of the sensitivity of the position and velocity constraints. It is assumed here that the sensitivities of the coefficients $\alpha$ and $\beta$, with respect to the design variables, are null.

\subsection{Sensitivities of the modes of vibration}

One of the weaknesses of automatic differentiation tools, pointed out by [33], concerns the reliability of the differential algorithm used for the solution of the eigenproblem, which is required when the mode component synthesis is used in the multibody formulation. Therefore, a closer look into the use of the results of the eigenproblem in the framework of flexible multibody systems is taken in this work, with the objective of devising a strategy to overcome the deficiency of the differentiated code to calculate the sensitivities of the modes of vibration and natural frequencies. The closed-form of the sensitivities of the eigenproblem is implemented directly to replace the automatic differentiated code.

The sensitivity of the nodal displacement vector is obtained by computing the derivative of Equation (11) with respect to the design variables as

$$
\frac{\mathrm{d} \mathbf{u}^{\prime}}{\mathrm{d} \mathbf{b}}=\frac{\partial \mathbf{X}}{\partial \mathbf{b}} \mathbf{w}+\mathbf{X} \frac{\partial \mathbf{w}}{\partial \mathbf{b}}=\mathbf{X}_{\mathbf{b}} \mathbf{w}+\mathbf{X w}_{\mathbf{b}}
$$

where $\mathbf{X}_{\mathbf{b}}$ are the sensitivities of the eigenmodes with respect to the design variables. Equation (45) relates the modal sensitivities with the nodal sensitivities. Haftka and Gürdal [7] suggest two different approaches to evaluate this transformation: the fixed-mode approach, where the derivatives of vibration modes are neglected, and the updated-mode approach, where the derivatives of vibration modes are retained. The fixed-mode approach is computationally less expensive but the updatedmode approach can be more accurate. Therefore, the computation of the modal forces and the modal stiffness matrix derivatives can be obtained using either approach. The modal stiffness matrix derivative using the updated-mode approach is computed as

$$
\frac{\partial}{\partial \mathbf{b}}\left(\mathbf{X}^{\mathrm{T}} \mathbf{K}_{f f} \mathbf{X}\right)=\frac{\partial \mathbf{X}^{\mathrm{T}}}{\partial \mathbf{b}} \mathbf{K}_{f f} \mathbf{X}+\mathbf{X}^{\mathrm{T}} \frac{\partial \mathbf{K}_{f f}}{\partial \mathbf{b}} \mathbf{X}+\mathbf{X}^{\mathrm{T}} \mathbf{K}_{f f} \frac{\partial \mathbf{X}}{\partial \mathbf{b}}
$$

and when using the fixed-mode approach the sensitivity of the modal stiffness matrix is

$$
\frac{\partial}{\partial \mathbf{b}}\left(\mathbf{X}^{\mathrm{T}} \mathbf{K}_{f f} \mathbf{X}\right)=\mathbf{X}^{\mathrm{T}} \frac{\partial \mathbf{K}_{f f}}{\partial \mathbf{b}} \mathbf{X}
$$

It should be noted that although automatic differentiation is used to calculate the stiffness matrix sensitivities the problems that arise from having repeated eigenvalues are the same as when using direct implementations of analytic sensitivities. Further note that $\mathbf{X}^{\mathrm{T}}\left(\partial \mathbf{K}_{f f} / \partial \mathbf{b}\right) \mathbf{X}$ can be null even when $\left|\partial \mathbf{K}_{f f} / \partial \mathbf{b}\right| \neq 0$, for instance, when using the modes of vibration associated with rigid body motions.

The computation of the sensitivities of the eigenmodes can be evaluated using the Nelson scheme [36] for the case of distinct eigenvalues. However, when repeated eigenvalues occur the 


\section{SENSITIVITY ANALYSIS OF FLEXIBLE MULTIBODY SYSTEMS}

sensitivities of $\mathbf{X}$ do not exist, only the directional derivatives exist, and Ojavo's method is used [37]. Ojavo's method is implemented and used in this work, instead of the differentiated part of the code that would 'solve' the eigenproblem [38].

\subsection{Derivative of the stiffness matrix of the plate/shell element}

In any application of the methodology proposed here the design variables related to the flexible elements may include the cross-sectional areas, plate thickness, moments of inertia, location of kinematic constraints or any material or geometric properties of these elements. However, for the sake of simplicity the only design variables considered henceforth are the layer orientations of the laminates, which are grouped in vector $\boldsymbol{\theta}$. In what follows it is assumed that the vector of design variables $\mathbf{b}$ coincides with the vector of layer orientations.

The stiffness matrix of the plate/shell element is described in Equation (18). In this equation only the sub-matrix $\mathbf{D}$ depends on the design variables. Thus, the sensitivity of the element stiffness matrix relative to the design variables is given by

$$
\frac{\partial \mathbf{K}_{f f}^{(e)}}{\partial \boldsymbol{\theta}}=\int_{0}^{1} \int_{0}^{1-\eta}\left(\mathbf{B}^{\mathrm{T}} \frac{\partial \mathbf{D}}{\partial \boldsymbol{\theta}} \mathbf{B}\right)^{(e)}|\mathbf{J}| \mathrm{d} \xi \mathrm{d} \eta
$$

Taking into account that $\mathbf{D}$ is the elasticity matrix, which is a function of the sub-matrices $\mathbf{D}_{m}, \mathbf{D}_{b}, \mathbf{D}_{m b}$ and $\mathbf{D}_{s}$ defined by Equation (20), its partial derivatives are

$$
\begin{aligned}
\left(\mathbf{D}_{m}, \mathbf{D}_{b}, \mathbf{D}_{m b}, \mathbf{D}_{s}\right)_{\boldsymbol{\theta}}= & \left(\sum_{k=1}^{n}\left(\mathbf{D}_{m}, \mathbf{D}_{b}, \mathbf{D}_{m b}, \mathbf{D}_{s}\right)_{k}\right)_{\boldsymbol{\theta}} \\
= & \sum_{k=1}^{n}\left(\left[\begin{array}{lll}
c_{11} & c_{12} & c_{16} \\
c_{21} & c_{22} & c_{26} \\
c_{61} & c_{62} & c_{66}
\end{array}\right]_{\boldsymbol{\theta}} H_{1},\left[\begin{array}{lll}
c_{11} & c_{12} & c_{16} \\
c_{21} & c_{22} & c_{26} \\
c_{61} & c_{62} & c_{66}
\end{array}\right]_{\boldsymbol{\theta}} H_{2},\right. \\
& {\left.\left[\begin{array}{lll}
c_{11} & c_{12} & c_{16} \\
c_{21} & c_{22} & c_{26} \\
c_{61} & c_{62} & c_{66}
\end{array}\right]_{\boldsymbol{\theta}} H_{3},\left[\begin{array}{ll}
c_{44} & c_{45} \\
c_{54} & c_{55}
\end{array}\right]_{\boldsymbol{\theta}}\right)_{k} }
\end{aligned}
$$

The evaluation of the partial derivatives of each of the sub-matrices presented in Equation (49) requires the computation of the partial derivative of Equation (15) with respect to the design variables, which is given by

$$
\left(\mathbf{C}_{\boldsymbol{\theta}}\right)_{k}=\left(\frac{\partial \mathbf{T}^{\mathrm{T}}}{\partial \boldsymbol{\theta}} \overline{\mathbf{C}} \mathbf{T}+\mathbf{T}^{\mathrm{T}} \overline{\mathbf{C}} \frac{\partial \mathbf{T}}{\partial \boldsymbol{\theta}}\right)_{k}
$$

for the $k$ th layer of the laminate. Note that the sensitivities in Equation (50) need to be evaluated only for the layers that have orientations defined as design variables.

The mass matrix of the plate/shell element does not depend on the design variables and, therefore, its partial derivative with respect to the design variable is null. 


\subsection{Derivative of the stiffness matrix of the beam element}

The stiffness matrix of the beam element is described by Equation (23). For this element only the elasticity matrix $\mathbf{D}$ depends on the design variables. The partial derivative of the beam stiffness matrix with respect to the design variables is given by

$$
\frac{\partial \mathbf{K}_{f f}^{(e)}}{\partial \boldsymbol{\theta}}=\int_{-1}^{1}\left(\mathbf{B}^{\mathrm{T}} \frac{\partial \mathbf{D}}{\partial \boldsymbol{\theta}} \mathbf{B}\right)^{(e)}|\mathbf{J}| \mathrm{d} \xi
$$

The partial derivative of the D matrix is obtained by using the code VABS, which was extended to consider this computation. In the Timoshenko theory matrix $\mathbf{D}$ is expressed by Equation (24) [26]. The partial derivative of the elastic matrix $\mathbf{D}$ with respect to the design variables is given by

$$
\frac{\partial \mathbf{D}}{\partial \boldsymbol{\theta}}=\left[\begin{array}{cc}
\frac{\partial \mathbf{X}}{\partial \boldsymbol{\theta}} & \frac{\partial \mathbf{F}}{\partial \boldsymbol{\theta}} \\
\left(\frac{\partial \mathbf{F}}{\partial \boldsymbol{\theta}}\right)^{\mathrm{T}} & \frac{\partial \mathbf{G}}{\partial \boldsymbol{\theta}}
\end{array}\right]
$$

The sub-matrices included in Equation (52) require the evaluation of the derivatives of the warping functions. These are obtained by the derivation of Equations (27) and (28), leading to

$$
\begin{aligned}
\frac{\partial \mathbf{V}_{0}}{\partial \boldsymbol{\theta}} & =\frac{\partial \hat{\mathbf{V}}_{0}}{\partial \boldsymbol{\theta}} \boldsymbol{\varepsilon}+\hat{\mathbf{V}}_{0} \frac{\partial \boldsymbol{\varepsilon}}{\partial \boldsymbol{\theta}} \\
\mathbf{E} \frac{\partial \hat{\mathbf{V}}_{0}}{\partial \boldsymbol{\theta}} & =\left(\mathbf{H} \boldsymbol{\psi}_{c l} \boldsymbol{\psi}_{c l}^{\mathrm{T}}-\mathbf{I}\right) \frac{\partial \mathbf{C}_{h \varepsilon}}{\partial \boldsymbol{\theta}}-\frac{\partial \mathbf{E}}{\partial \boldsymbol{\theta}} \hat{\mathbf{V}}_{0}
\end{aligned}
$$

and

$$
\begin{aligned}
\frac{\partial \mathbf{V}_{1}}{\partial \boldsymbol{\theta}} & =\frac{\partial \hat{\mathbf{V}}_{1}}{\partial \boldsymbol{\theta}} \boldsymbol{\varepsilon}^{\prime}+\hat{\mathbf{V}}_{1} \frac{\partial \boldsymbol{\varepsilon}^{\prime}}{\partial \boldsymbol{\theta}} \\
\mathbf{E} \frac{\partial \hat{\mathbf{V}}_{1}}{\partial \boldsymbol{\theta}} & =\left(\mathbf{I}-\mathbf{H} \boldsymbol{\psi}_{c l} \boldsymbol{\psi}_{c l}^{\mathrm{T}}\right) \frac{\partial\left[\mathbf{C}_{\varepsilon l}^{\mathrm{T}}+\left(\mathbf{C}_{h l}^{\mathrm{T}}-\mathbf{C}_{h l}\right) \hat{\mathbf{V}}_{0}\right]}{\partial \boldsymbol{\theta}}-\frac{\partial \mathbf{E}}{\partial \boldsymbol{\theta}} \hat{\mathbf{V}}_{1}
\end{aligned}
$$

The terms obtained in Equations (53) and (54) are required to calculate the derivatives of matrices $\mathbf{X}, \mathbf{F}$ and $\mathbf{G}$ in Equation (52). Taking into account Equations (29)-(34) the following derivative is obtained:

$$
\frac{\partial \mathbf{G}^{-1}}{\partial \boldsymbol{\theta}}=\mathbf{Q}^{\mathrm{T}} \frac{\partial \mathbf{O}^{-\mathrm{T}}}{\partial \boldsymbol{\theta}} \mathbf{R} \mathbf{O}^{-1} \mathbf{Q}+\mathbf{Q}^{\mathrm{T}} \mathbf{O}^{-\mathrm{T}} \frac{\partial \mathbf{R}}{\partial \boldsymbol{\theta}} \mathbf{O}^{-1} \mathbf{Q}+\mathbf{Q}^{\mathrm{T}} \mathbf{O}^{-\mathrm{T}} \mathbf{R} \frac{\partial \mathbf{O}^{-1}}{\partial \boldsymbol{\theta}} \mathbf{Q}
$$

where the derivatives of $\mathbf{O}$ and $\mathbf{R}$ are obtained as

$$
\begin{aligned}
\frac{\partial \mathbf{O}}{\partial \boldsymbol{\theta}}= & \frac{\partial \mathbf{X}}{\partial \boldsymbol{\theta}}-\frac{\partial\left(\mathbf{F G}^{-1} \mathbf{F}^{\mathrm{T}}\right)}{\partial \boldsymbol{\theta}} \\
\frac{\partial \mathbf{R}}{\partial \boldsymbol{\theta}}= & \frac{\partial \hat{\mathbf{V}}_{0}^{\mathrm{T}}}{\partial \boldsymbol{\theta}} \mathbf{C}_{l l} \hat{\mathbf{V}}_{0}+\hat{\mathbf{V}}_{0}^{\mathrm{T}} \frac{\partial \mathbf{C}_{l l}}{\partial \boldsymbol{\theta}} \hat{\mathbf{V}}_{0}+\hat{\mathbf{V}}_{0}^{\mathrm{T}} \mathbf{C}_{l l} \frac{\partial \hat{\mathbf{V}}_{0}}{\partial \boldsymbol{\theta}}+\frac{\partial \hat{\mathbf{V}}_{1}^{\mathrm{T}}}{\partial \boldsymbol{\theta}}\left(\mathbf{C}_{h l}+\mathbf{C}_{h l}^{\mathrm{T}}\right) \hat{\mathbf{V}}_{0} \\
& +\hat{\mathbf{V}}_{1}^{\mathrm{T}} \frac{\partial\left(\mathbf{C}_{h l}+\mathbf{C}_{h l}^{\mathrm{T}}\right)}{\partial \boldsymbol{\theta}} \hat{\mathbf{V}}_{0}+\hat{\mathbf{V}}_{1}^{\mathrm{T}}\left(\mathbf{C}_{h l}+\mathbf{C}_{h l}^{\mathrm{T}}\right) \frac{\partial \hat{\mathbf{V}}_{0}}{\partial \boldsymbol{\theta}}+\frac{\partial \hat{\mathbf{V}}_{1}^{\mathrm{T}}}{\partial \boldsymbol{\theta}} \mathbf{C}_{l \varepsilon}+\hat{\mathbf{V}}_{1}^{\mathrm{T}} \frac{\partial \mathbf{C}_{l \varepsilon}}{\partial \boldsymbol{\theta}}
\end{aligned}
$$


and matrix $\partial \mathbf{O}^{-1} / \partial \boldsymbol{\theta}$ is computed using [39]

$$
\frac{\partial \mathbf{O}^{-1}}{\partial \boldsymbol{\theta}}=-\mathbf{O}^{-1} \frac{\partial \mathbf{O}}{\partial \boldsymbol{\theta}} \mathbf{O}^{-1}
$$

The derivative of matrix $\mathbf{F}$, required in Equation (52), is expressed as

$$
\frac{\partial \mathbf{F}}{\partial \boldsymbol{\theta}}=\frac{\partial \mathbf{B}^{\mathrm{T}}}{\partial \boldsymbol{\theta}} \mathbf{A}^{-1} \mathbf{Q G}+\mathbf{B}^{\mathrm{T}} \frac{\partial \mathbf{A}^{-1}}{\partial \boldsymbol{\theta}} \mathbf{Q G}+\mathbf{B}^{\mathrm{T}} \mathbf{A}^{-1} \mathbf{Q} \frac{\partial \mathbf{G}}{\partial \boldsymbol{\theta}}
$$

the derivative of matrix $\mathbf{B}$ being given by

$$
\frac{\partial \mathbf{B}}{\partial \boldsymbol{\theta}}=\left(\hat{\mathbf{V}}_{0}^{\mathrm{T}} \mathbf{C}_{h l}+\mathbf{C}_{\varepsilon l}^{\mathrm{T}}\right) \frac{\partial \hat{\mathbf{V}}_{0}}{\partial \boldsymbol{\theta}}+\frac{\partial\left(\hat{\mathbf{V}}_{0}^{\mathrm{T}} \mathbf{C}_{h l}+\mathbf{C}_{\varepsilon l}^{\mathrm{T}}\right)}{\partial \boldsymbol{\theta}} \hat{\mathbf{V}}_{0}
$$

and the derivative of matrix $\mathbf{A}$ by

$$
\frac{\partial \mathbf{A}}{\partial \boldsymbol{\theta}}=\frac{\partial\left(\mathbf{C}_{\varepsilon \varepsilon}+\hat{\mathbf{V}}_{0}^{\mathrm{T}} \mathbf{C}_{h \varepsilon}\right)}{\partial \boldsymbol{\theta}}
$$

The derivatives of $\mathbf{X}$ is given by

$$
\frac{\partial \mathbf{X}}{\partial \boldsymbol{\theta}}=\frac{\partial \mathbf{A}}{\partial \boldsymbol{\theta}}+\frac{\partial \mathbf{F}}{\partial \boldsymbol{\theta}} \mathbf{G}^{-1} \mathbf{F}^{\mathrm{T}}+\mathbf{F} \frac{\partial \mathbf{G}^{-1}}{\partial \boldsymbol{\theta}} \mathbf{F}^{\mathrm{T}}+\mathbf{F} \mathbf{G}^{-1} \frac{\partial \mathbf{F}^{\mathrm{T}}}{\partial \boldsymbol{\theta}}
$$

It should be noted that the automatic differentiation of the quantities used by the beam element requires the availability of the source code VABS [26]. When the VABS code is not available or when the reader suspects that computational advantages can be obtained from the direct implementation of the analytic sensitivities, it is rather simple to code Equations (52)-(60), if only layer orientations are used as design variables.

\section{DEMONSTRATIVE EXAMPLES}

A slider-crank mechanism with a flexible connected rod, reported by Dias and Pereira [40], is used here to demonstrate the procedures proposed in this work. In the first model of the mechanism the rod is modeled with a composite beam element, whereas in the second model composite/shell finite elements are used to represent the coupler. The properties and the sensitivities of the cross section of the rod with respect to the layer orientation, for the first model, are computed by Equations (24) and (52), respectively, whereas the stiffness matrix of the rod of the slider-crank of the second model and its sensitivities are obtained using Equations (18) and (49), respectively.

\subsection{Rod modeled with composite beam elements}

The mechanism shown in Figure 4 consists of a slider-crank with the same dimensions of the model presented by Dias and Pereira [40]. However, the material used in the rod is a composite with the properties $E_{1}=128 \mathrm{GPa}, E_{2}=E_{3}=10.1 \mathrm{GPa}, G_{12}=G_{13}=G_{23}=5.36 \mathrm{GPa}, v=0.23$ and $\rho=1586 \mathrm{~kg} / \mathrm{m}^{3}$. The beam is a tube, with a hollow circular cross section, made of composite materials, with an area of $3.16 \times 10^{-5} \mathrm{~m}^{2}$, for which the layer fiber orientations are depicted in 


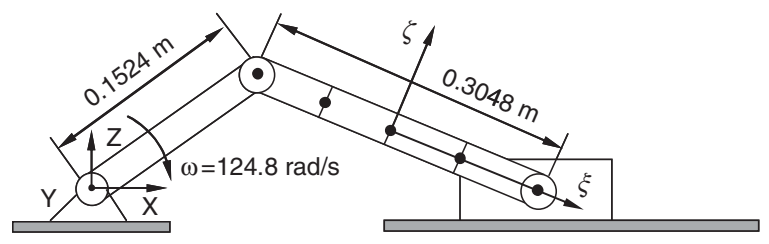

Figure 4. Slider-crank mechanism with a composite beam.

Table I. Characteristics of the three lay-ups used to model the composite beam.

\begin{tabular}{lccc}
\hline Lay-ups & 1 & 2 & 3 \\
Layer orientation & $0^{\circ}$ & $15^{\circ}$ & $30^{\circ}$ \\
\hline
\end{tabular}

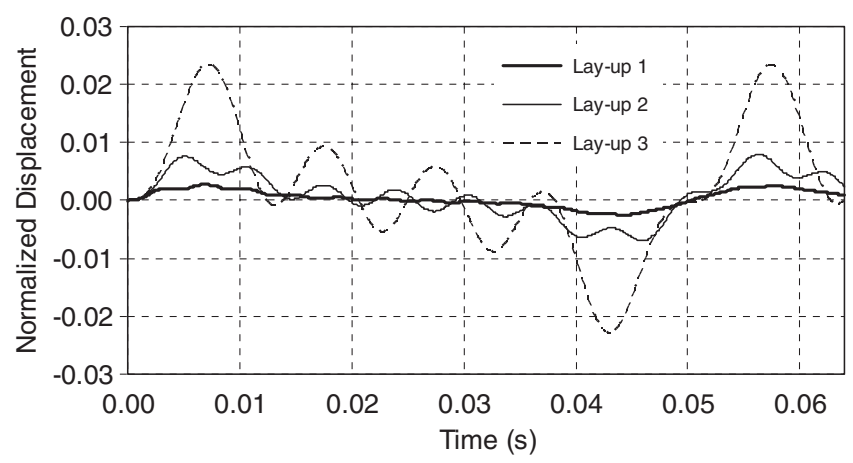

Figure 5. Dimensionless mid-node rod displacement.

Table I. Four modes of vibration are used to reduce the number of generalized elastic coordinates of the mechanism, according to the methodology presented in this work.

The model of the flexible body includes proportional damping such that 1 and $3 \%$ of modal damping is used for the 1st and 4th natural frequencies, respectively [41]. The simulation results for the model, in terms of a normalized displacement, defined as the ratio of the connecting beam mid-node $\zeta$-deflection to the beam length, are presented in Figure 5.

Owing to the different beam stiffnesses resulting from the different layer arrangements in the various lay-ups, the displacements of the models are not similar. The layer orientations of lay-ups 2 and 3 have been selected as design variables. In Figure 6 the analytic sensitivities, obtained by using the automatic differentiated code, and numerical sensitivities of the normalized displacement of the mid-node of the rod are plotted. Note that the validity of the results presented requires that the assumptions for small beam deformations be fulfilled, which is the case in all lay-ups discussed here.

For lay-up 3, the analytic sensitivities and the forward difference sensitivities computed for perturbation of the design variable of $1 \%$ are coincident during the period of analysis used. For lay-up 2, the analytic and numerical sensitivities, with a perturbation of the design variable of $1 \%$, 


\section{SENSITIVITY ANALYSIS OF FLEXIBLE MULTIBODY SYSTEMS}
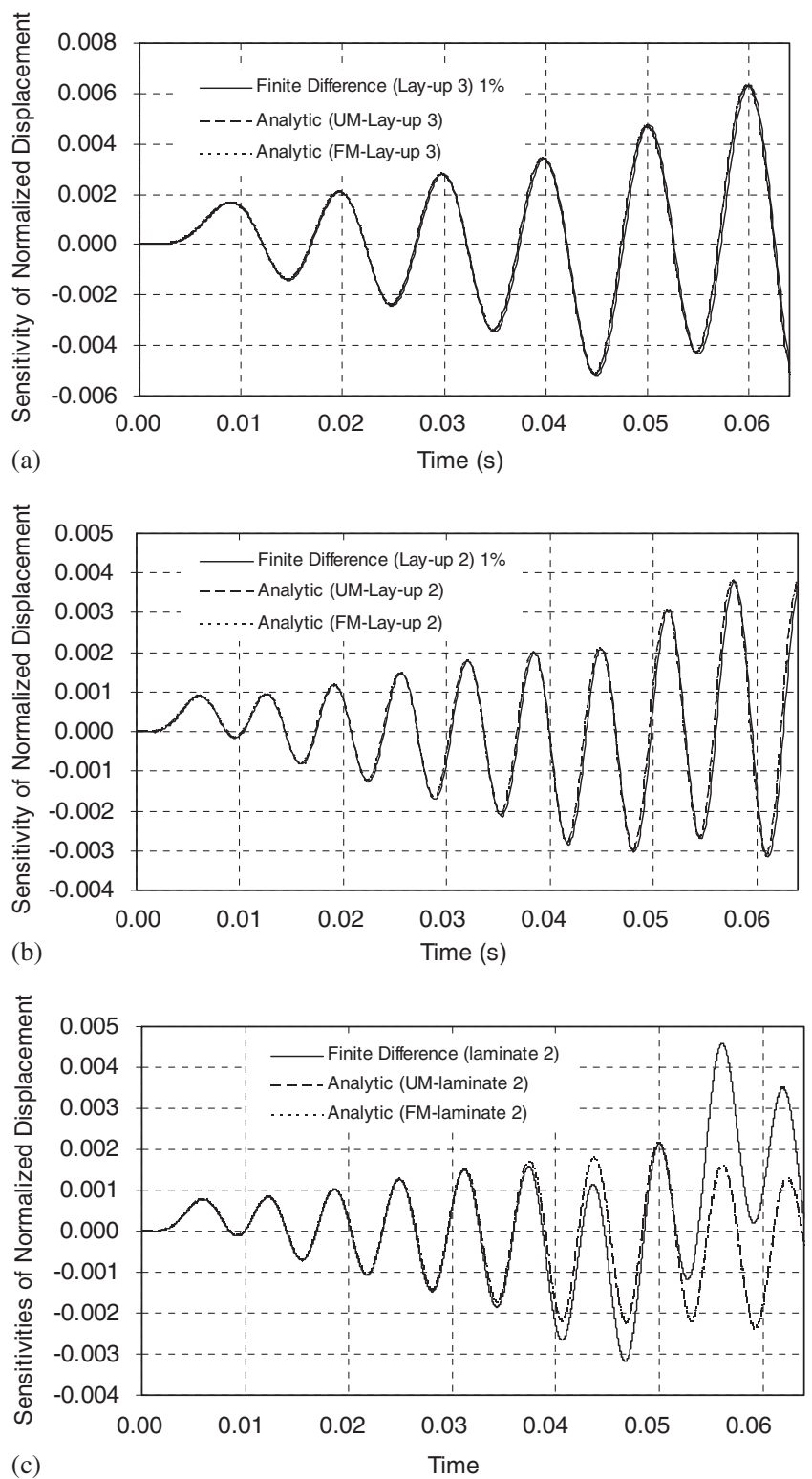

Figure 6. Sensitivities of the mid-node rod displacement with respect to the layer orientation: (a) lay-up 3; (b) lay-up 2 with fixed step integration; and (c) lay-up 2 with variable step integration.

are coincident, during all the period of simulation, when a small constant time step is used for the integrator, as shown in Figure 6(b). However, when a variable time step integrator is used, the numerical sensitivities start diverging from the analytical sensitivities, as shown in Figure 6(c). The problems of evaluating numerical sensitivities with variable time step integrators have been pointed 
out by several authors [42]. Removing the design variable dependency of the time step used in the integration algorithm is important to the accuracy and to the stability computation of the finite difference sensitivities. When constant time-step integration algorithms are used, the accuracy of the response requires the use of small time steps [43]. The optimal time step size for the proper evaluation of finite difference sensitivities is estimated by Haftka and Malkus [44]. However, the use of variable time step integration is not only common in the framework of multibody dynamics but also unavoidable for most of the systems modeled, with all the consequences that it has in the accuracy of the numerical sensitivities.

The example presented shows that for particular arrangements of the flexible multibody model the numerical and analytic sensitivities may lead to similar results, but there is no guarantee that such equivalence holds even for small variations of the model. Certainly this observation can be used as a word of warning when using numerical sensitivities in the framework of optimization flexible multibody systems. In any case, for the application case presented no major differences are observed when using the updated- and the fixed-mode approach for the calculation of the modes of vibration of the flexible body.

\subsection{Rod modeled with composite plate elements}

The second application consists of the slider-crank previously used, but in which the flexible coupler has a rectangular cross section. The coupler is modeled by composite plates with a thickness of $0.0075 \mathrm{~m}$ and a total depth of $0.04 \mathrm{~m}$, as shown in Figure 7. In this model the mid surface of the plate is the plane $\xi \eta$ and the material used has the same properties of the beam model. The orientations of the layers for the different lay-ups considered are defined in Table II. This model uses proportional damping for the connecting rod and the damping coefficients are chosen in such a way that the modal damping is $1 \%$ for the 1 st natural frequency and $3 \%$ for the 6 th natural frequency.

The simulation results are presented in Figure 8 in terms of the dimensionless displacement defined as the ratio of the $\zeta$-deflection of the mid-node of the connecting plate to the plate length. The layers' orientations, for the different lay-ups, are selected as design variables. It should be noted that a necessary condition for the validity of the results is that, for each lay-up considered,

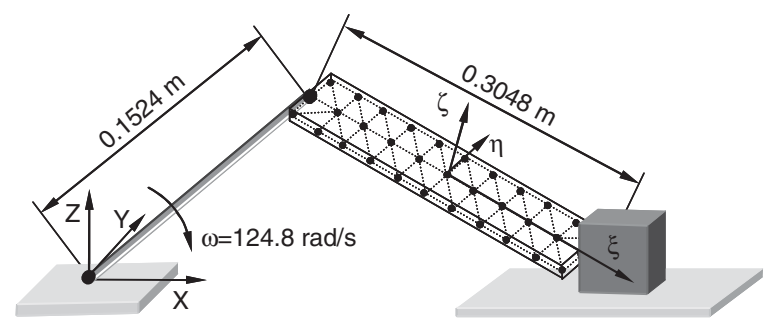

Figure 7. Slider-crank mechanism with a composite plate.

Table II. Characteristics of the five lay-ups used to model the composite plate.

\begin{tabular}{lccccc}
\hline Lay-ups & 1 & 2 & 3 & 4 & 5 \\
Layer orientation & $0^{\circ}$ & $15^{\circ}$ & $30^{\circ}$ & $75^{\circ}$ & $90^{\circ}$ \\
\hline
\end{tabular}




\section{SENSITIVITY ANALYSIS OF FLEXIBLE MULTIBODY SYSTEMS}

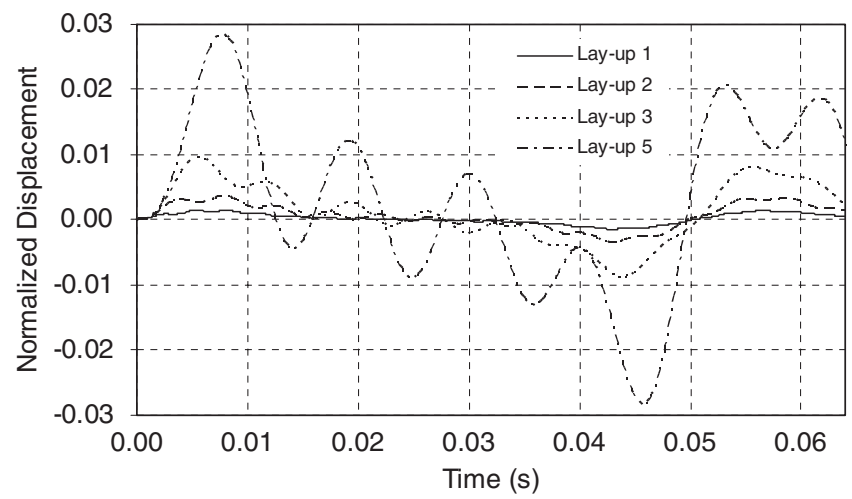

Figure 8. Dimensionless mid-node $\zeta$-displacement of the rod for the several lay-ups.

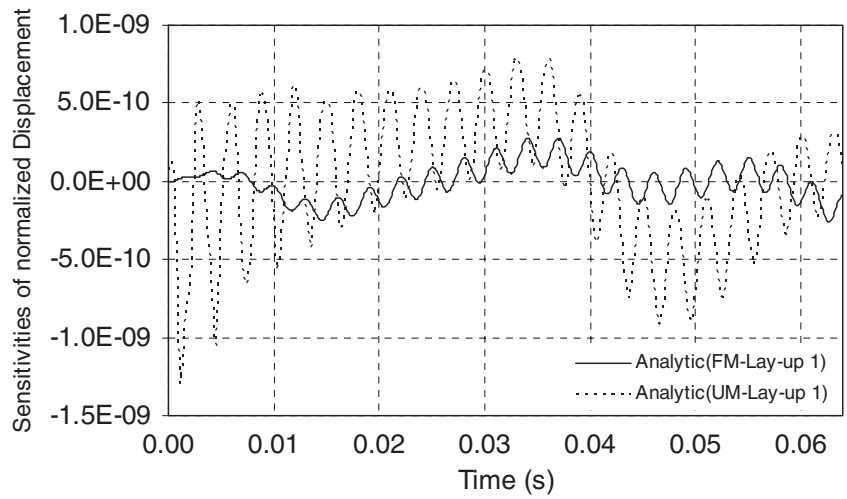

Figure 9. Analytic sensitivities of the connecting rod mid-node $\zeta$-displacement using lay-up 1.

only small deformations are observed. Any lay-up for which the small deformation assumption is not valid must be discarded from any further consideration, even if the sensitivity analysis is used in the framework of optimization. In this case, constraints on the maximum allowable deflection of the connecting rod must be set.

In Figures 9 and 10 the analytic sensitivities of the normalized displacement of the midnode of the plate are plotted for lay-up 1 and lay-up 5, respectively, using the fixed- and the updated-mode approaches. The results emphasize that although for some lay-ups the updated-and fixed-mode approaches lead to similar results, in other configurations, major differences between the sensitivities obtained with these methods exist. Therefore, the use of fixed-mode approach for flexible multibody systems with composite materials, in the framework of sensitivity analysis or of optimization, is not always reliable.

In order to emphasize the numerical difficulty associated with the evaluation of the numerical sensitivities, the forward difference results are presented for several variations in the layer orientations in Figures 11-13. The size of the variations used to calculate the forward differences is limited to be larger than 0.005 because the condition errors increase too much afterwards. The selection 


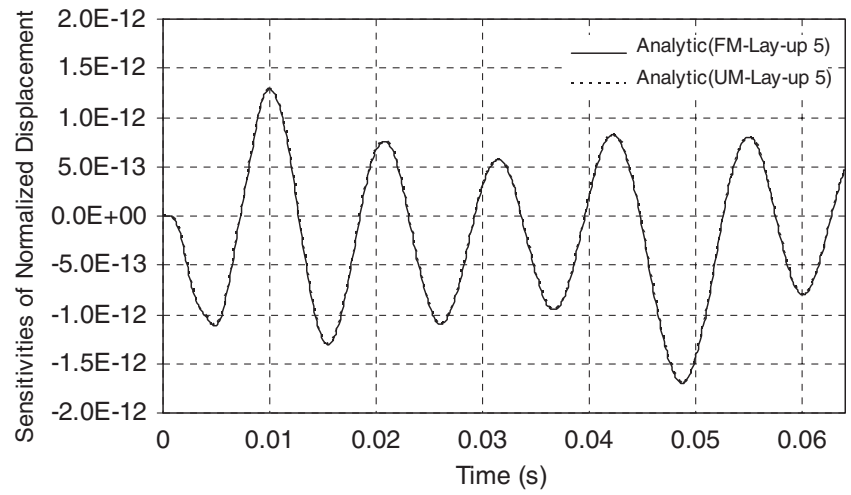

Figure 10. Analytic sensitivity of the connecting rod mid-node $\zeta$-displacement for lay-up 5.

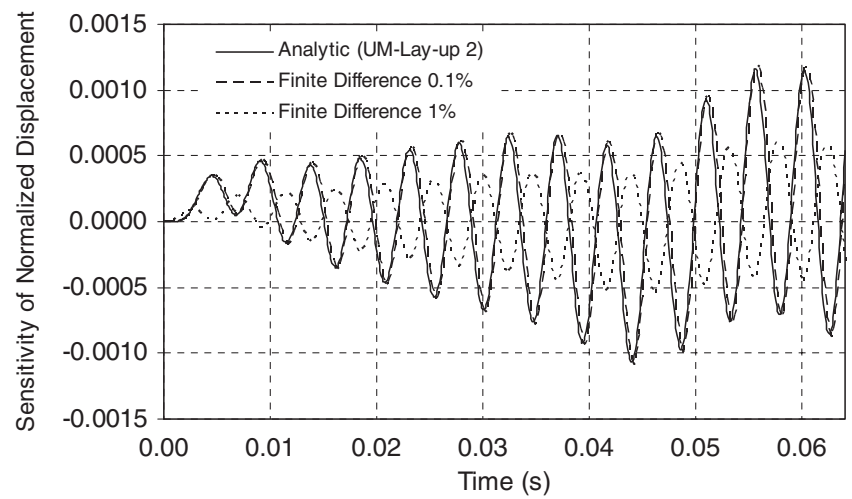

Figure 11. Analytical and numerical finite difference sensitivities of the connecting rod mid-node displacement with lay-up 2.

of a large variation also leads to errors in the calculation of the derivatives due to the truncation of some terms in the approximation series. Therefore, in all that follows only variations between 0.5 and $10 \%$ are used in the calculation of the finite differences.

Figure 11 shows that for lay-up 2 the forward finite difference sensitivities computed with a perturbation of $0.1 \%$ are almost coincident with the analytic sensitivities. However, perturbing the layer orientation of lay-up 3 by $0.1 \%$ to compute the finite difference sensitivities does not give the convergence of results as for the case of lay-up 2. In fact, Figure 12 shows that the convergence between the finite difference and the analytic sensitivities is assured only if the finite difference sensitivities are computed for perturbations of the design variable close to $5 \%$. Although not shown in Figure 12, perturbations in the range of $0.5-3 \%$ lead to numerical sensitivities completely different from the analytical sensitivities. For lay-up 4 the forward finite difference sensitivities computed with a perturbation of $1 \%$ led to values similar to the analytic sensitivities, as shown in Figure 13.

The results show that, as expected, the selection of the finite difference variation has a large influence on the sensitivities obtained when using the forward difference method being the similarity 


\section{SENSITIVITY ANALYSIS OF FLEXIBLE MULTIBODY SYSTEMS}

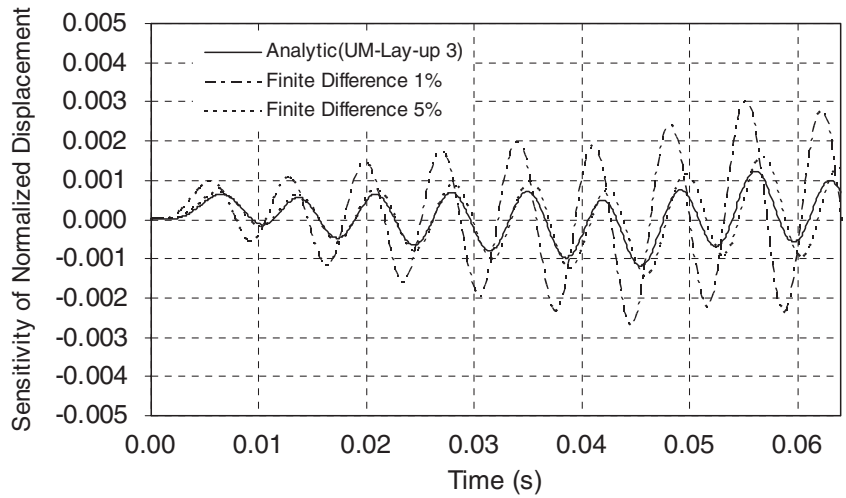

Figure 12. Analytical and numerical finite difference sensitivities of the connecting rod mid-node displacement with lay-up 3.

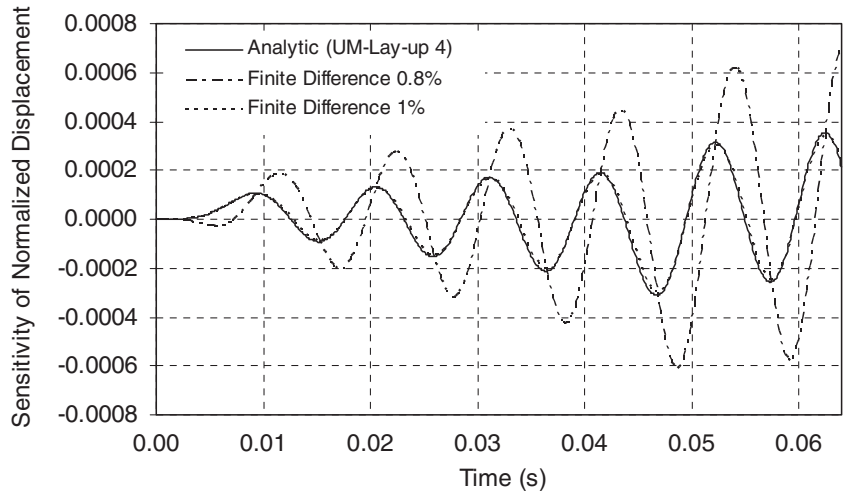

Figure 13. Analytical and numerical finite difference sensitivities of the connecting rod mid-node displacement with lay-up 4.

with the analytic sensitivities dependent on the lay-up considered and on the finite difference variation. Although some of the divergence between the analytical and numerical sensitivities can be attributed to the use of a variable time step integrator, as shown in Section 5.1, the fact remains that for a general flexible multibody application it is difficult, if not impossible, to find a variation for the numeric sensitivities accurate for all. Moreover, due to the need of using variable time step integrators in multibody codes, it may lead to a dependency of the design variable on the time step, which affects accuracy, and to the stability computation of the finite difference sensitivities.

\section{CONCLUSIONS}

A general method to obtain the analytical sensitivities of flexible multibody systems made of composite materials by automatic differentiation was presented in this work. The equations of motion of a flexible multibody system and of the relevant finite elements used when modeling 
composite materials were introduced. The derivation of the analytic sensitivities was demonstrated, the properties of the lamina of the composites being used as design variables. It was shown that the analytical sensitivities of the system response with respect to the selected design variables are rather complex equations requiring a reasonable implementation effort. By using two simple flexible multibody systems made of composite materials and comparing their sensitivities, obtained by the methodology proposed and by the numerical differences, it was possible to confirm that the analytic sensitivities lead to more robust solutions for all cases of material arrangements of a composite structure. It was shown that the numerical sensitivities may have accuracy similar to the analytical sensitivities by controlling the size of the variations and removing their dependency on the variation of the time step of the numerical integrator. In the process, the correct implementation of the analytical sensitivities obtained with the automatic differentiation tool was demonstrated. Eventually, the use of higher-order or adaptive sensitivities would also lead to very accurate numerical sensitivities. However, no general rule can be applied for all numerical sensitivities in a general purpose flexible multibody code. The application cases presented in this work also showed that the use of the updated-mode approach is preferred to the use of the fixed-mode approach when calculating the sensitivities of the modes of vibration. When the sensitivities are to be used in the framework of optimization problems where the iterative procedures lead to different lay-up arrangements for the lamina, methods that lead to less reliable results should be avoided. The use of automatic differentiation tools is demonstrated to be a valuable alternative to the traditional encoding of the sensitivities. However, the use of automatic differentiation cannot be done as a black box, because it not only implies major changes in the sequence of the computations associated with the analysis of the system but also requires decisions on which equations should be obtained symbolically and which must be implemented analytically.

\section{ACKNOWLEDGEMENTS}

The source code of VABS was made available by Profs D. Hodges, W. Yu and O. Bauchau for this work. The authors express their gratitude for the code and for the discussions that followed its implementation and use.

\section{REFERENCES}

1. Gerstmayr J, Ambrósio J. Component mode synthesis with constant mass and stiffness matrices applied to flexible multibody systems. International Journal for Numerical Methods in Engineering 2007; DOI: 10.1002/nme.2133.

2. Kövecses J, Angeles J. The stiffness matrix in elastically articulated rigid-body systems. Multibody System Dynamics 2007; 18(2):169-184.

3. Bauchau O, Wang J. Stability evaluation and system identification of flexible multibody systems. Multibody System Dynamics 2007; 18(1):95-106.

4. Lehner M, Eberhard P. On the use of moment-matching to build reduced order models in flexible multibody dynamics. Multibody System Dynamics 2006; 17(2):191-211.

5. Adelman HM, Haftka RT. Sensitivity analysis of discrete structural systems. AIAA Journal 1986; 24:823-832.

6. Greene W, Haftka R. Computational aspects of sensitivity calculations in transient structural analysis. Computers and Structures 1989; 32(2):433-443.

7. Haftka R, Gürdal Z. Elements of Structural Optimization. Kluwer Academic Publishers: Dordrecht, The Netherlands, 1992.

8. Dias J. Análise de Sensibilidades e Optimização de Sistemas Mecânicos Rígido-Flexíveis (Sensitivity analysis and optimization of rigid-flexible mechanical systems). Ph.D. Dissertation, Instituto Superior Técnico de Lisboa, Lisboa, Portugal, 1999. 


\section{SENSITIVITY ANALYSIS OF FLEXIBLE MULTIBODY SYSTEMS}

9. Bischof C, Carle A, Corliss G, Griewank A, Hovland P. ADIFOR: generating derivative codes from Fortran programs. Scientific Programming 1992; 1(1):11-29.

10. Bischof C, Carle A, Khademi P, Mauer A. ADIFOR 2.0: automatic differentiation of Fortran 77 programs. IEEE Computational Science and Engineering 1996; 3(3):18-32.

11. Griewank A, Reese S. On the calculation of Jacobian matrices by the Markowitz rule. In Proceedings of the Automatic Differentiation of Algorithms: Theory, Implementation, and Applications, Griewank A, Corliss G (eds). SIAM: Philadelphia, PA, 1991; 126-135.

12. Eberhard P. Analysis and optimization of complex multibody systems using advanced sensitivity analysis methods. Proceedings of the ICIAM/GAMM, Hamburg, 1995. Zeitschrift fur Angewandte Mathematik und Mechanik 1996; 76(3):40-43.

13. Cook R, Malkus D, Plesha M. Concepts and Applications of Finite Elements Analysis. Wiley: New York, 1989.

14. Gonçalves J, Ambrósio J. Advanced modeling of flexible multibody dynamics using virtual bodies. Computer Assisted Mechanics and Engineering Sciences 2002; 9(3):373-390.

15. Ambrósio J, Gonçalves J. Complex flexible multibody systems with application to vehicle dynamics. Multibody Systems Dynamics 2001; 6(2):163-182.

16. Anderson E, Bai Z, Bischof C, Blackford S, Demmel J, Dongarra J, Du Croz J, Greenbaum A, Hammarling S, Mckenney A, Sorensen D. LAPACK Users' Guide. SIAM: Philadelphia, PA, 1999.

17. Baumgarte J. Stabilization of constraints and integrals of motion. Computer Methods in Applied Mechanics and Engineering 1972; 1:1-66.

18. Nikravesh PE. Computer-aided Analysis of Mechanical Systems. Prentice-Hall: Englewood Cliffs, NJ, 1988.

19. Neto MA, Ambrósio J. Stabilization methods for the integration of DAE in the presence of redundant constraints. Multibody System Dynamics 2003; 10:81-105.

20. Yoo W, Haug E. Dynamics of flexible mechanical systems using vibration and static correction modes. Journal of Mechanisms, Transmissions and Automation in Design 1986; 108:315-322.

21. Pereira M, Proença P. Dynamic analysis of spatial flexible multibody systems using joint co-ordinates. International Journal for Numerical Methods in Engineering 1991; 32:1799-1812.

22. Reddy JN. Mechanics of Laminated Composite Plates: Theory and Analysis. CRC Press: Boca Raton, FL, 1997.

23. Carrera E. $C^{0}$ Reissner-Mindlin multilayered plate elements including zig-zag and interlaminar stress continuity. International Journal for Numerical Methods in Engineering 1996; 39:1797-1820.

24. Batoz J, Lardeur P. A discrete shear triangular nine d.o.f. element for the analysis of thick to very thin plates. International Journal for Numerical Methods in Engineering 1989; 28:533-560.

25. Oñate E. Cálculo de Estructuras por el Método de Elementos Finitos (5 Segunda Edicion). Centro Internacional de Métodos Numéricos en Ingenieria: Barcelona, Spain, 1995.

26. Yu W, Hodges DH, Volovoi V, Cesnik CES. On Timoshenko-like modeling of initially curved and twisted composite beams. International Journal of Solids and Structures 2002; 39:5101-5121.

27. Popescu B, Hodges D. On asymptotically correct Timoshenko-like anisotropic beam theory. International Journal of Solids and Structures 2000; 37:535-558.

28. Cesnik C, Hodges D. VABS: a new concept for composite rotor blade cross-sectional modeling. Journal of the American Helicopter Society 1997; 42:27-38.

29. Samin JC, Brüls O, Collard JF, Sass L, Fisette P. Multiphysics modeling and optimization of mechatronic multibody systems. Multibody System Dynamics 2007; 18(3):345-373.

30. Leboeuf F, Bessonnet G, Seguin P, Lacouture P. Energetic versus sthenic optimality criteria for gymnastic movement synthesis. Multibody System Dynamics 2006; 17(3):213-236.

31. Bottasso CL, Prilutsky BI, Croce A, Imberti E, Sartirana S. A numerical procedure for inferring from experimental data the optimization cost functions using a multibody model of the neuro-musculoskeletal system. Multibody System Dynamics 2006; 17(2):123-154.

32. Cai G-P, Lim CW. Optimal tracking control of a flexible hub-beam system with time delay. Multibody System Dynamics 2006; 17(4):331-350.

33. Eberhard P, Bischof C. Automatic differentiation of numerical integration algorithms. Mathematics of Computation 1999; 68:717-731.

34. Shampine L, Gordon M. Computer Solution of Ordinary Differential Equations. Freeman: San Francisco, CA, 1975.

35. IMSL: Fortran Routines for Mathematical Applications. Visual Numerics Inc.: Houston, TX, 1997.

36. Nelson R. Simplified calculation of eigenvector derivatives. AIAA Journal 1976; 14(9):1201-1205.

37. Dailey L. Eigenvector derivatives with repeated eigenvalues. AIAA Journal 1989; 27(4):486-491.

Copyright (C) 2008 John Wiley \& Sons, Ltd.

Int. J. Numer. Meth. Engng (2008)

DOI: $10.1002 / \mathrm{nme}$ 
38. Neto MA. Optimization of flexible multibody systems with composite materials. Ph.D. Dissertation, University of Coimbra, Coimbra, Portugal, 2006.

39. Leal A. Elementos Mistos em Projecto Óptimo de Estruturas. Ph.D. Dissertation, Departamento de Engenharia Mecânica, Faculdade de Ciências e Tecnologia da Universidade de Coimbra, Coimbra, Portugal, 1992.

40. Dias J, Pereira M. Sensitivity analysis of rigid-flexible multibody systems. Multibody System Dynamics 1997; 1:303-322.

41. Ambrósio J, Ravn P. Elastodynamics of multibody systems using generalized inertial coordinates and structural damping. Mechanics of Structures and Machines 1997; 25:201-219.

42. van KA, Haftka RT, Kim NH. Review of options for structural design sensitivity analysis. Part I: linear systems. Computer Methods in Applied Mechanics and Engineering 2005; 194:3213-3243.

43. Cho S, Choi KK. Design sensitivity analysis and optimization of nonlinear transient dynamics. International Journal for Numerical Methods in Engineering 2002; 48:351-373.

44. Haftka R, Malkus DS. Calculation of sensitivity derivatives in thermal problems by finite differences. International Journal for Numerical Methods in Engineering 1981; 17:1811-1821. 